\title{
On the logarithmic comparison theorem for integrable logarithmic connections*
}

\author{
F. J. Calderón Moreno and L. Narváez Macarro
}

\begin{abstract}
Let $X$ be a complex analytic manifold, $D \subset X$ a free divisor with jacobian ideal of linear type (e.g. a locally quasi-homogeneous free divisor), $j: U=X-D \hookrightarrow X$ the corresponding open inclusion, $\mathcal{E}$ an integrable logarithmic connection with respect to $D$ and $\mathcal{L}$ the local system of the horizontal sections of $\mathcal{E}$ on $U$. In this paper we prove that the canonical morphisms

$$
\Omega_{X}^{\bullet}(\log D)(\mathcal{E}(k D)) \rightarrow R j_{*} \mathcal{L}, \quad j_{!} \mathcal{L} \rightarrow \Omega_{X}^{\bullet}(\log D)(\mathcal{E}(-k D))
$$

are isomorphisms in the derived category of sheaves of complex vector spaces for $k \gg 0$ (locally on $X$ ).
\end{abstract}

MSC: 32C 38; 14F40; 32S40

\section{Introduction}

Let $X$ be a $n$-dimensional complex analytic manifold. An ideal $\mathcal{I} \subset \mathcal{O}_{X}$ is said to be of linear type if the canonical homomorphism from its symmetric algebra to its Rees algebra is an isomorphism. We say that a divisor (=hypersurface) $D \subset X$ is of linear jacobian type if its Jacobian ideal is of linear type.

This paper is devoted to prove the following result (see Corollaries (3.2.7), $(3.2 .8)$ :

Let $D \subset X$ be a free divisor of linear jacobian type, $j: U=X-D \hookrightarrow X$ the corresponding open inclusion, $\mathcal{E}$ an integrable logarithmic connection with respect to $D$ and $\mathcal{L}$ the local system of the horizontal sections of $\mathcal{E}$ on $U$. Then, for any point $p \in D$ there is an open neighborhood $V$ of $p$ and

\footnotetext{
${ }^{*}$ The authors are partially supported by MTM2004-07203-C02-01 and FEDER.
} 
an integer $k_{0}$ such that, for $k \geq k_{0}$, the restrictions to $V$ of the canonical morphisms

$$
\Omega_{X}^{\bullet}(\log D)(\mathcal{E}(k D)) \rightarrow R j_{*} \mathcal{L}, \quad j_{!} \mathcal{L} \rightarrow \Omega_{X}^{\bullet}(\log D)(\mathcal{E}(-k D))
$$

are isomorphisms in the derived category of sheaves of complex vector spaces.

Since any locally quasi-homogeneous free divisor is of linear jacobian type, the above result generalizes the logarithmic comparison theorem proved in [1] for the case $\mathcal{E}=\mathcal{O}_{X}$ and in [16], II, $\S 6$, and [17], Appendix A, for normal crossing divisors.

Let us note that the Gauss-Manin construction associated with versal unfoldings of hypersurface singularities produces non-trivial examples of integrable logarithmic connections (with respect to the discriminant) satisfying our hypothesis (cf. [40, 1]).

Our methods are based on $D$-module theory and on our previous results in [5, 7, 8]. See also [12, 44, 13] for related work.

Let us now comment on the content of this paper.

In section 1 we introduce the notations that we will use throughout the paper and we recall some notions and basic results on Lie-Rinehart algebras, free divisors, the Bernstein construction and the Koszul property. We also recall and refine some results in [7, and focus on the linear type properties for a free divisor and the facts that any locally quasi-homogeneous free divisors is of linear jacobian type and that any free divisor of linear jacobian type is Koszul free.

In section 2 we give an improved version of our characterization theorem in [8] of the logarithmic comparison problem for logarithmic integrable connections. By the way, we deduce a new and short proof of the logarithmic comparison theorem for the trivial connection in [11].

In section 3 we state and prove the main results of this paper. Namely, a "parametric" comparison theorem between logarithmic and usual BernsteinKashiwara modules associated with integrable logarithmic connections (see Theorem (3.1.1) and Corollary (3.1.2) , and the logarithmic comparison theorem for integrable logarithmic connections, both with respect to free divisors of linear jacobian type.

In section 4 we apply the above results to describe algebraically "intersection $D$-modules". Namely, given an integrable logarithmic connection $\mathcal{E}$ with respect to a free divisor of linear jacobian type $D \subset X$, we describe 
in terms of $\mathcal{E}$ the regular holonomic $D$-module which corresponds, via the Riemann-Hilbert correspondence of Mebkhout-Kashiwara, to the intersection complex of Deligne-Goresky-MacPherson associated with the local system of horizontal sections of $\mathcal{E}$ on $X-D$.

We thank T. Torrelli for useful comments on a previous version on this paper.

\section{Notations and preliminary results}

Let $X$ be a $n$-dimensional complex analytic manifold and $D \subset X$ a hypersurface (= divisor), and let us denote by $j: U=X-D \hookrightarrow X$ the corresponding open inclusion. We denote by $\pi: T^{*} X \rightarrow X$ the cotangent bundle, $\mathcal{O}_{X}$ the sheaf of holomorphic functions on $X, \mathcal{D}_{X}$ the sheaf of linear differential operators on $X$ (with holomorphic coefficients), $\operatorname{Gr} \mathcal{D}_{X}$ the graded ring associated with the filtration $F$ by the order and $\sigma(P)$ the principal symbol of a differential operator $P$. If $J \subset \mathcal{D}_{X}$ is a left ideal, we denote by $\sigma(J)$ the corresponding graded ideal of $\operatorname{Gr} \mathcal{D}_{X}$.

Let us denote by $\operatorname{Jac}(D) \subset \mathcal{O}_{X}$ the Jacobian ideal of $D \subset X$, i.e. the coherent ideal of $\mathcal{O}_{X}$ whose stalk at any $p \in X$ is the ideal generated by $h, \frac{\partial h}{\partial x_{1}}, \ldots, \frac{\partial h}{\partial x_{n}}$, where $h \in \mathcal{O}_{X, p}$ is any reduced local equation of $D$ at $p$ and $x_{1}, \ldots, x_{n} \in \mathcal{O}_{X, p}$ is a system of local coordinates centered at $p$.

We say that $D$ is quasi-homogeneous at $p \in D$ if there is a system of local coordinates $\underline{x}$ centered at $p$ such that the germ $(D, p)$ has a reduced weighted homogeneous defining equation (with strictly positive weights) with respect to $\underline{x}$. We say that $D$ is locally quasi-homogeneous if it is so at each point $p \in D$.

For any bounded complex $\mathcal{K}$ of sheaves of $\mathbb{C}$-vector spaces on $X$, let us denote by $\mathcal{K}^{\vee}=R \operatorname{Hom}_{\mathbb{C}_{X}}\left(\mathcal{K}, \mathbb{C}_{X}\right)$ its Verdier dual.

If $A$ is a commutative ring and $M$ an $A$-module, we will denote by $\operatorname{Sym}_{A}(M)$ its symmetric algebra. If $I \subset A$ is an ideal, we will denote by $\mathcal{R}(I)=\oplus_{d=0}^{\infty} I^{d} t^{d} \subset A[t]$ its Rees algebra.

\subsection{Lie-Rinehart algebras}

Let $k \rightarrow A$ be a homomorphism of commutative rings.

Let us denote by $\operatorname{Der}_{k}(A)$ the $A$-module of $k$-linear derivations $\delta: A \rightarrow$ $A$. It is a left sub- $A$-module of $\operatorname{End}_{k}(A)$ closed by the bracket [-, - ]. If $\delta, \delta^{\prime} \in \operatorname{Der}_{k}(A)$ and $a \in A$ we have $\left[\delta, a \delta^{\prime}\right]=a\left[\delta, \delta^{\prime}\right]+\delta(a) \delta^{\prime}$.

(1.1.1) Definition. (Cf. [18, 37, 39]) A Lie-Rinehart algebra over $(k, A)$, or a $(k, A)$-Lie algebra, is an $A$-module $L$ endowed with a $k$-Lie algebra structure 
and an $A$-linear map $\rho: L \rightarrow \operatorname{Der}_{k}(A)$, called "anchor map", which is also a morphism of Lie algebras and satisfies

$$
\left[\lambda, a \lambda^{\prime}\right]=a\left[\lambda, \lambda^{\prime}\right]+\rho(\lambda)(a) \lambda^{\prime}
$$

for $\lambda, \lambda^{\prime} \in L$ and $a \in A$.

In order to simplify, we write $\lambda(a) \stackrel{\text { not. }}{=} \rho(\lambda)(a)$ for $\lambda \in L$ and $a \in A$.

(1.1.2) Example. 1) The first example of Lie-Rinehart algebra is $L=$ $\operatorname{Der}_{k}(A)$ with the identity as anchor morphism.

2) More generally, for any ideal $I \subset A$, the set

$$
\operatorname{Der}_{k}(\log I):=\left\{\delta \in \operatorname{Der}_{k}(A) \mid \delta(I) \subset I\right\}
$$

is a sub- $A$-module and a sub-k-Lie algebra of $\operatorname{Der}_{k}(A)$ which becomes a LieRinehart algebra by considering the inclusion $\operatorname{Der}_{k}(\log I) \hookrightarrow \operatorname{Der}_{k}(A)$ as anchor map.

(1.1.3) Definition. Let $L, L^{\prime}$ be Lie-Rinehart algebras over $(k, A)$. A morphism of Lie-Rinehart algebras from $L$ to $L^{\prime}$ is an $A$-linear map $F: L \rightarrow$ $L^{\prime}$ which is a morphism of Lie algebras and satisfies $\lambda(a)=F(\lambda)(a), \forall \lambda \in L$, $\forall a \in A$.

(1.1.4) Definition. An $A$-ring is a (not necessarily commutative) ring $B$ with a ring homomorphism $\eta: A \rightarrow B$. We say that the $A$-ring $(B, \eta)$ is central over $k$ if $\eta(c) b=b \eta(c)$ for any $b \in B$ and any $c \in k$.

(1.1.5) Definition. Let $L$ be a Lie-Rinehart algebra over $(k, A)$ and $R$ a $A$-ring central over $k$. We say that a $k$-linear map $\varphi: L \rightarrow R$ is admissible if:

a) $\varphi(a \lambda)=a \varphi(\lambda)$ for $\lambda \in L$ and $a \in A$, i.e. $\varphi$ is a morphism of left $A$-modules,

b) $\varphi\left(\left[\lambda, \lambda^{\prime}\right]\right)=\left[\varphi(\lambda), \varphi\left(\lambda^{\prime}\right)\right]$ for $\lambda, \lambda^{\prime} \in L$, i.e. $\varphi$ is a morphism of Lie algebras,

c) $\varphi(\lambda) a-a \varphi(\lambda)=\lambda(a) 1_{R}$ for $\lambda \in L$ and $a \in A$.

(1.1.6) Theorem. ([39]) For any Lie-Rinehart algebra $L$ over $(k, A)$ there exists an A-ring $U$, central over $k$, and an admissible map $\theta: L \rightarrow U$ which are universal in the sense that, for any $A$-ring $R$ central over $k$ and any admissible map $\varphi: L \rightarrow R$, there exists a unique A-ring homomorphism $h: U \rightarrow R$ such that $h \circ \theta=\varphi$. 
The pair $(U, \theta)$ in the above theorem is clearly unique, up to a unique isomorphism. It is called the enveloping algebra of $L$ and it is denoted by $\mathrm{U}(L)$. Some authors call $\mathrm{U}(L)$ the universal algebra of $L$ (cf. [19]).

The algebra $\mathrm{U}(L)$ has a natural filtration $F^{\bullet}$ given by the powers of the image of $\theta$. If $L$ is a projective $A$-module, the Poincaré-Birkhoff-Witt theorem [39] asserts that its associated graded ring is canonically isomorphic to the symmetric algebra of the $A$-module $L$, and so the map $\theta$ is injective.

For any (commutative) scalar extension $k \rightarrow k^{\prime}$ and any Lie-Rinehart algebra $L$ over $(k, A), k^{\prime} \otimes_{k} L$ inherits an obvious Lie-Rinehart algebra structure over $\left(k^{\prime}, k^{\prime} \otimes_{k} A\right)$.

(1.1.7) From the universal property of $\mathrm{U}(L)$, any left $\mathrm{U}(L)$-module $M$ is determined by the admissible map

$$
\lambda \in L \mapsto[m \mapsto \lambda m] \in \operatorname{End}_{k}(M) .
$$

Let us suppose for now that $L$ is a projective $A$-module of finite rank, and let us consider the dual $A$-module $\Omega_{L}:=\operatorname{Hom}_{A}(L, A)$ and the "exterior differential"

$$
d: A \rightarrow \Omega_{L}, \quad(d a)(\lambda)=\lambda(a), \quad a \in A .
$$

The map (1) is so uniquely determined by the connection

$$
\nabla: M \rightarrow \Omega_{L} \otimes_{A} M, \quad \nabla(m)(\lambda)=\lambda m, \quad m \in M, \lambda \in L,
$$

where we have identified $\Omega_{L} \otimes_{A} M=\operatorname{Hom}_{A}(L, M)$. The connection $\nabla$ satisfies the Leibniz rule $\nabla(a m)=a \nabla(m)+(d a) \otimes m$ and the admissibility of the map (1) is equivalent to the integrability condition on $\nabla$ in the usual sense cf. [16], I, 2.14.

(1.1.8) The Cartan-Eilenberg-Chevalley-Rinehart-Spencer complexes (cf. [15, 10, 39, 38, 28, 22])

In the following, let us suppose that $L \subset L^{\prime}$ is a pair of Lie-Rinehart algebras over $(k, A)$ and $E$ is a left $\mathrm{U}(L)$-module.

The Cartan-Eilenberg-Chevalley-Rinehart-Spencer complex associated with $\left(L, L^{\prime}, E\right)$ is the complex $\operatorname{Sp}_{L, L^{\prime}}(E)$ defined by $\operatorname{Sp}_{L, L^{\prime}}^{-r}(E)=\mathrm{U}\left(L^{\prime}\right) \otimes_{A}\left(\bigwedge^{r} L\right) \otimes_{A}$ 
$E, r \geq 0$ and the differential $\varepsilon^{-r}: \operatorname{Sp}_{L, L^{\prime}}^{-r}(E) \rightarrow \operatorname{Sp}_{L, L^{\prime}}^{-(r-1)}(E)$ is given by:

$$
\begin{gathered}
\varepsilon^{-r}\left(P \otimes\left(\lambda_{1} \wedge \cdots \wedge \lambda_{r}\right) \otimes e\right)= \\
=\sum_{i=1}^{r}(-1)^{i-1}\left(P \lambda_{i}\right) \otimes\left(\lambda_{1} \wedge \cdots \wedge \widehat{\lambda_{i}} \wedge \cdots \wedge \lambda_{r}\right) \otimes e- \\
-\sum_{i=1}^{r}(-1)^{i-1} P \otimes\left(\lambda_{1} \wedge \cdots \wedge \widehat{\lambda_{i}} \wedge \cdots \wedge \lambda_{r}\right) \otimes\left(\lambda_{i} e\right)+ \\
+\sum_{1 \leq i<j \leq r}(-1)^{i+j} P \otimes\left(\left[\lambda_{i}, \lambda_{j}\right] \wedge \lambda_{1} \wedge \cdots \wedge \widehat{\lambda_{i}} \wedge \cdots \wedge \widehat{\lambda_{j}} \wedge \cdots \wedge \lambda_{r}\right) \otimes e
\end{gathered}
$$

for $r \geq 2$, and $\varepsilon^{-1}\left(P \otimes \lambda_{1} \otimes e\right)=\left(P \lambda_{1}\right) \otimes e-P \otimes\left(\lambda_{1} e\right)$ for $r=1$, and $P \in \mathrm{U}\left(L^{\prime}\right), \lambda_{i} \in L, e \in E$.

We also have an obvious natural augmentation

$$
\varepsilon^{0}: \operatorname{Sp}_{L, L^{\prime}}^{0}(E)=\mathrm{U}\left(L^{\prime}\right) \otimes_{A} E \rightarrow h^{0}\left(\operatorname{Sp}_{L, L^{\prime}}(E)\right)=\mathrm{U}\left(L^{\prime}\right) \otimes_{\mathrm{U}(L)} E .
$$

We write $\operatorname{Sp}_{L, L^{\prime}}=\operatorname{Sp}_{L, L^{\prime}}(A)$ and $\operatorname{Sp}_{L}(E)=\operatorname{Sp}_{L, L}(E)$. Let us note that $\operatorname{Sp}_{L, L}=\operatorname{Sp}_{L}(A)$ and

$$
\operatorname{Sp}_{L, L^{\prime \prime}}(E)=\mathrm{U}\left(L^{\prime \prime}\right) \otimes_{\mathrm{U}\left(L^{\prime}\right)} \operatorname{Sp}_{L, L^{\prime}}(E)
$$

for $L^{\prime \prime} \supset L^{\prime}$ a third Lie-Rinehart algebra over $(k, A)$.

(1.1.9) Proposition. Let us suppose that $L$ is a projective A-module of finite rank and that $E$ is a left $\mathrm{U}(L)$-module, flat (resp. projective) over $A$. Then the complex $\operatorname{Sp}_{L}(E)$ is a finite $\mathrm{U}(L)$-resolution (resp. a finite projective $\mathrm{U}(L)$-resolution) of $E$. Moreover, if $L$ and $E$ are free $A$-modules, then $\operatorname{Sp}_{L}(E)$ is a finite free $\mathrm{U}(L)$-resolution of $E$.

Proof. We proceed as in [8], p. 52. We consider the filtration on the augmented complex $\operatorname{Sp}_{L}(E) \rightarrow E$ given by

$$
F^{i} \operatorname{Sp}_{L}^{-k}(E)=\left(F^{i-k} \mathrm{U}(L)\right) \otimes_{A} \bigwedge^{k} L \otimes_{A} E, \quad F^{i} E=E, \quad i \geq 0 .
$$

Its graded complex is, by using the Poincaré-Birkhoff-Witt theorem, canonically isomorphic to the tensor product by $-\otimes_{A} E$ of the augmented complex

$$
\operatorname{Sym}_{A}(L) \otimes_{A} \dot{\bigwedge} L \stackrel{d^{0}}{\rightarrow} A,
$$

where the differential is given by

$$
d^{-k}\left(P \otimes\left(\lambda_{1} \wedge \cdots \wedge \lambda_{k}\right)\right)=\sum_{i=1}^{k}(-1)^{i-1}\left(P \lambda_{i}\right) \otimes\left(\lambda_{1} \wedge \cdots \wedge \widehat{\lambda_{i}} \wedge \cdots \wedge \lambda_{k}\right)
$$


for $P \in \operatorname{Sym}_{A}(L), \lambda_{1}, \ldots, \lambda_{k} \in L$ and $k=1, \ldots, \mathrm{rk}_{A} L$, and

$$
d^{0}: \operatorname{Sym}_{A}(L) \otimes_{A} \bigwedge^{0} L=\operatorname{Sym}_{A}(L) \rightarrow A
$$

is the obvious augmentation. The proposition follows from the exactness of (44) (cf. [4], $§ 9,3)$ and the flatness of $E$.

\section{(1.1.10) Lie algebroids}

The notions and results above can be easily generalized to the case in which our ring homomorphism $k \rightarrow A$ is replaced by a morphism of sheaves of commutative rings $\mathcal{K} \rightarrow \mathcal{A}$ on a topological space, for instance when $X$ is a complex analytic manifold and we consider the morphism $\mathbb{C}_{X} \rightarrow \mathcal{O}_{X}$ or $\mathbb{C}_{X}[s] \rightarrow \mathcal{O}_{X}[s]$. In that case it is customary to talk about Lie algebroids instead of Lie-Rinehart algebras. If $\mathcal{L}$ is a Lie algebroid over $(\mathcal{K}, \mathcal{A})$, its stalk $\mathcal{L}_{p}$ at a point $p$ is a Lie-Rienhart algebra over $\left(\mathcal{K}_{p}, \mathcal{A}_{p}\right)$. We leave the reader to decide the details (see [24, 25] as general references for Lie algebroids on differentiable manifolds and [14] for the interplay between complex Lie algebroids and $\mathcal{D}$-module theory).

(1.1.11) Example. 1) The first example of Lie algebroid is $\mathcal{L}=\operatorname{Der}_{\mathbb{C}}\left(\mathcal{O}_{X}\right)$ with the identity as anchor morphism.

2) The sheaf of differential operators of order $\leq 1, F^{1} \mathcal{D}_{X}=\mathcal{O}_{X} \oplus \operatorname{Der}_{\mathbb{C}}\left(\mathcal{O}_{X}\right)$, with the projection $F^{1} \mathcal{D}_{X} \rightarrow \operatorname{Der}_{\mathbb{C}}\left(\mathcal{O}_{X}\right)$ as anchor morphism, is a Lie algebroid.

3) Any submodule $\mathcal{L} \subset \operatorname{Der}_{\mathbb{C}}\left(\mathcal{O}_{X}\right)$ which is closed for the bracket is a Lie algebroid with the inclusion as anchor morphism. This applies in particular to $\mathcal{L}=\operatorname{Der}(\log D)=\{\log a r i t h m i c$ vector fields with respect to $D\}$ [41.

\subsection{Logarithmic derivations and free divisors}

We say that $D$ is a free divisor [41] if the $\mathcal{O}_{X}$-module $\operatorname{Der}(\log D)$ of logarithmic vector fields with respect to $D$ is locally free (of rank $n$ ), or equivalently if the $\mathcal{O}_{X}$-module $\Omega_{X}^{1}(\log D)$ of logarithmic 1 -forms with respect to $D$ is locally free (of rank $n$ ).

Normal crossing divisors, plane curves, free hyperplane arrangements (e.g. the union of reflecting hyperplanes of a complex reflection group), discriminant of left-right stable mappings or bifurcation sets are example of free divisors.

Let us denote by $\mathcal{D}_{X}(\log D)$ the 0-term of the Malgrange-Kashiwara filtration with respect to $D$ on the sheaf $\mathcal{D}_{X}$ of linear differential operators 
on $X$ (cf. [26], Def 4.1-1). When $D$ is a free divisor, the first author has proved in [5] that $\mathcal{D}_{X}(\log D)$ is the universal enveloping algebra of the Lie algebroid $\operatorname{Der}(\log D)$, and so it is coherent and it has noetherian stalks of finite global homological dimension. Locally, if $\left\{\delta_{1}, \ldots, \delta_{n}\right\}$ is a local basis of the logarithmic vector fields on an open set $V$, any differential operator in $\Gamma\left(V, \mathcal{D}_{X}(\log D)\right)$ can be written in a unique way as a finite sum

$$
\sum_{\substack{\alpha \in \mathbb{N}^{n} \\|\alpha| \leq d}} a_{\alpha} \delta_{1}^{\alpha_{1}} \cdots \delta_{n}^{\alpha_{n}}
$$

where the $a_{\alpha}$ are holomorphic functions on $V$.

\subsection{The ring $\mathcal{D}[s]$ and the Bernstein construction}

Let $p$ be a point in $D$ and $f \in \mathcal{O}=\mathcal{O}_{X, p}$ a reduced local equation of $D$. Let us write $\mathcal{D}=\mathcal{D}_{X, p}$.

On the polynomial ring $\mathcal{D}[s]$, with $s$ a central variable, there are two natural filtrations: the filtration induced by the order filtration on $\mathcal{D}$, that we also denote by $F$, and the total order filtration $F_{T}$ given by

$$
F_{T}^{k} \mathcal{D}[s]=\sum_{i=0}^{k}\left(F^{i} \mathcal{D}\right) s^{k-i}, \quad \forall k \geq 0 .
$$

For each $P \in \mathcal{D}[s]$ let us denote by $\sigma(P)$ (resp. $\sigma_{T}(P)$ ) its principal symbol in $\operatorname{Gr}_{F} \mathcal{D}[s]$ (resp. in $\operatorname{Gr}_{F_{T}} \mathcal{D}[s]$ ).

The filtered ring $(\mathcal{D}[s], F)$ is the ring of $\mathbb{C}[s]$-linear differential operators with coefficient in $\mathcal{O}[s]$ and so it is the enveloping algebra of the Lie-Rinehart algebra $\operatorname{Der}_{\mathbb{C}[s]}(\mathcal{O}[s])=\mathbb{C}[s] \otimes_{\mathbb{C}} \operatorname{Der}_{\mathbb{C}}(\mathcal{O})$ over $(\mathbb{C}[s], \mathcal{O}[s])$, whereas the filtered ring $\left(\mathcal{D}[s], F_{T}\right)$ is the enveloping algebra of the Lie-Rinehart algebra $F^{1} \mathcal{D}=$ $\mathcal{O} \oplus \operatorname{Der}_{\mathbb{C}}(\mathcal{O})$ over $(\mathbb{C}, \mathcal{O})$ whose anchor map is the projection $\mathcal{O} \oplus \operatorname{Der}_{\mathbb{C}}(\mathcal{O}) \rightarrow$ $\operatorname{Der}_{\mathbb{C}}(\mathcal{O})$. In the latter case the canonical map $F^{1} \mathcal{D} \rightarrow \mathcal{D}[s]$ sends every $a \in \mathcal{O}$ to as.

The canonical maps

$$
\eta: \operatorname{Sym}_{\mathcal{O}[s]}\left(\operatorname{Der}_{\mathbb{C}[s]}(\mathcal{O}[s])\right) \rightarrow \operatorname{Gr}_{F} \mathcal{D}[s], \quad \eta_{T}: \operatorname{Sym}_{\mathcal{O}}\left(F^{1} \mathcal{D}\right) \rightarrow \operatorname{Gr}_{F_{T}} \mathcal{D}[s]
$$

are isomorphisms of graded $\mathcal{O}[s]$-algebras and $\mathcal{O}$-algebras respectively.

The free module of rank one over the ring $\mathcal{O}\left[f^{-1}, s\right]$ generated by the symbol $f^{s}, \mathcal{O}\left[f^{-1}, s\right] f^{s}$, has a natural left module structure over the ring $\mathcal{D}[s]$ : the action of a derivation $\delta \in \operatorname{Der}_{\mathbb{C}}(\mathcal{O})$ is given by $\delta\left(f^{s}\right)=\delta(f) s f^{-1} f^{s}$ (see [3]). 
Let us call $\operatorname{Jac}(f)=\operatorname{Jac}(D)_{p}$ the Jacobian ideal of $f$,

$$
\varphi_{0}: \operatorname{Sym}_{\mathcal{O}}\left(F^{1} \mathcal{D}\right) \rightarrow \mathcal{R}(\operatorname{Jac}(f)) \subset \mathcal{O}[t]
$$

the composition of the canonical surjective map $\operatorname{Sym}_{\mathcal{O}} \operatorname{Jac}(f) \rightarrow \mathcal{R}(\operatorname{Jac}(f))$ with the surjective map $\operatorname{Sym}_{\mathcal{O}}\left(F^{1} \mathcal{D}\right) \rightarrow \operatorname{Sym}_{\mathcal{O}}(\operatorname{Jac}(f))$ induced by

$$
P \in F^{1} \mathcal{D} \mapsto P(f) \in \operatorname{Jac}(f),
$$

and

$$
\varphi:=\varphi_{0} \circ \eta_{T}^{-1}: \operatorname{Gr}_{F_{T}} \mathcal{D}[s] \rightarrow \mathcal{R}(\operatorname{Jac}(f))
$$

For each $P \in \mathcal{D}[s]$ of total order $d$, we have that $P\left(f^{s}\right)=Q(s) f^{-d} f^{s}$ where $Q(s)$ is a polynomial of degree $d$ in $s$ with coefficients in $\mathcal{O}$. Let us call $C_{P, d} \in \mathcal{O}$ the highest coefficient of $Q(s)$.

The following lemma is well-known and the proof is straightforward (cf. [46], chap. I, Prop. 2.3).

(1.3.1) Lemma. With the above notations, we have $\varphi\left(\sigma_{T}(P)\right)=C_{P, d} t^{d}$ and so $\sigma_{T}\left(\operatorname{ann}_{\mathcal{D}[s]} f^{s}\right) \subset \operatorname{ker} \varphi$.

It is clear that $F_{T}^{0} \operatorname{ann}_{\mathcal{D}[s]} f^{s}=0$ and that

$$
\Theta_{f, s}:=F_{T}^{1} \operatorname{ann}_{\mathcal{D}[s]} f^{s}
$$

is formed by the operators $\delta-\alpha s$ with $\delta \in \operatorname{Der}_{\mathbb{C}}(\mathcal{O}), \alpha \in \mathcal{O}$ and $\delta(f)=\alpha f$. One easily sees that the $\mathcal{O}$-linear map

$$
\delta \in \operatorname{Der}(\log D)_{p} \mapsto \delta-\frac{\delta(f)}{f} s \in \Theta_{f, s}
$$

is an isomorphism of Lie-Rinehart algebras over $(\mathbb{C}, \mathcal{O})$. We obtain a canonical isomorphism

$$
\Theta_{f, s} \simeq \operatorname{Gr}_{F_{T}}^{1} \operatorname{ann}_{\mathcal{D}[s]} f^{s} .
$$

On the other hand, the homogeneous part of degree one $[\operatorname{ker} \varphi]_{1} \subset \operatorname{ker} \varphi$ is also canonically isomorphic to $\Theta_{f, s}$, and so we obtain

$$
\operatorname{Gr}_{F_{T}}^{1} \operatorname{ann}_{\mathcal{D}[s]} f^{s}\left(=\left[\sigma_{T}\left(\operatorname{ann}_{\mathcal{D}[s]} f^{s}\right)\right]_{1}=\sigma_{T}\left(\Theta_{f, s}\right)\right)=[\operatorname{ker} \varphi]_{1}
$$

\subsection{Divisors of linear type}

(1.4.1) Definition. (Cf. [45], \$7.2) Let $A$ be a commutative ring and $I \subset A$ an ideal. We say that $I$ is of linear type if the canonical (surjective) map of graded $A$-algebras $\operatorname{Sym}_{A}(I) \rightarrow \mathcal{R}(I)$ is an isomorphism. 
Ideals generated by a regular sequence are the first example of ideals of linear type.

(1.4.2) Definition. (see also [42]) We say that the divisor $D$ is of linear jacobian type at $p \in D$ if the stalk at $p$ of its jacobian ideal is of linear type. We say that $D$ is of linear jacobian type if it is so at any $p \in D$.

(1.4.3) Remark. To say that $D$ is of linear jacobian type at $p$ is equivalent to saying that $\operatorname{ker} \varphi$ (see ([6) ) is generated by its homogeneous part of degree $1,[\operatorname{ker} \varphi]_{1}=\sigma_{T}\left(\Theta_{f, s}\right)$.

Theorem 5.6 of [7] can be rephrased in the following way:

(1.4.4) Theorem. Any locally quasi-homogeneous free divisor is of linear jacobian type.

(1.4.5) Definition. Let $p \in D$ and let us write $\mathcal{O}=\mathcal{O}_{X, p}$ and $\mathcal{D}=\mathcal{D}_{X, p}$. We say that $D$ is of differential linear type at $p \in D$ if for some (or any, one easily sees that this condition does not depend on the choice of the local equation) reduced local equation $f \in \mathcal{O}$ of $D$ at $p$, the ideal $\operatorname{ann}_{\mathcal{D}[s]} f^{s}$ is generated by total order one operators, i.e. (see (17)) $\operatorname{ann}_{\mathcal{D}[s]} f^{s}=\mathcal{D}[s] \cdot \Theta_{f, s}$. We say that $D$ is of differential linear type if it is so at any $p \in D$.

It is clear that the set of points at which a divisor $D$ is of linear jacobian or differential linear type is open in $D$.

(1.4.6) Proposition. If the divisor $D$ is of linear jacobian type (at $p \in D$ ), then it is of differential linear type (at $p \in D$ ) and if $f \in \mathcal{O}_{X, p}$ is a reduced local equation of $D$ at $p$, then

$$
\operatorname{Gr}_{F_{T}} \operatorname{ann}_{\mathcal{D}[s]} f^{s}\left(=\sigma_{T}\left(\operatorname{ann}_{\mathcal{D}[s]} f^{s}\right)\right)=\operatorname{ker} \varphi .
$$

Proof. It is the same proof as those of Proposition 3.2 in [7], but here we consider $\operatorname{Gr}_{F_{T}} \operatorname{ann}_{\mathcal{D}[s]} f^{s}$ and the "true" jacobian ideal $\operatorname{Jac}(f)=\left(f, f_{x_{1}}^{\prime}, \ldots, f_{x_{n}}^{\prime}\right)$ instead of $\operatorname{Gr}_{F} \operatorname{ann}_{\mathcal{D}} f^{s}$ and $J_{f}=\left(f_{x_{1}}^{\prime}, \ldots, f_{x_{n}}^{\prime}\right)$.

\subsection{The Koszul property}

In this section, we fix a homomorphism of commutative rings $k \rightarrow A$ (resp. a homomorphism of sheaves of commutative rings $\mathcal{K} \rightarrow \mathcal{A}$ on a topological space $M)$ and all $(k, A)$-Lie-Rinehart algebras (resp. all Lie algebroids over $(\mathcal{K}, \mathcal{A}))$ will be free $A$-modules of finite rank (resp. locally free of finite rank over $\mathcal{A}$ ). 
We also assume that $D \subset X$ is a free divisor.

Let us recall that $D$ is a Koszul free divisor [5] at a point $p \in D$ if the symbols of any (or some) local basis $\left\{\delta_{1}, \ldots, \delta_{n}\right\}$ of $\operatorname{Der}(\log D)_{p}$ form a regular sequence in $\operatorname{Gr} \mathcal{D}_{X, p}$. We say that $D$ is a Koszul free divisor if it is so at any point $p \in D$. Actually, as M. Schulze pointed out, Koszul freeness is equivalent to holonomicity in the sense of [41].

Plane curves and locally quasi-homogeneous free divisors (e.g. free hyperplane arrangements or discriminant of left-right stable mappings in Mather's "nice dimensions") are example of Koszul free divisors [6].

(1.5.1) Definition. 1) Let $E \subset F$ be a pair of free $A$-modules of finite rank. We say that $(E, F)$ is a Koszul pair (over $A$ ) if some (or any) basis of $E$ forms a regular sequence in the symmetric algebra $\operatorname{Sym}_{A}(F)$.

2) Similarly, we say that a pair $(\mathcal{E}, \mathcal{F})$ of locally free $\mathcal{A}$-modules of finite rank, with $\mathcal{E} \subset \mathcal{F}$, is a Koszul pair (over $\mathcal{A}$ ) if $\left(\mathcal{E}_{p}, \mathcal{F}_{p}\right)$ is a Koszul pair over $\left(\mathcal{K}_{p}, \mathcal{L}_{p}\right)$ for any point $p \in M$.

To say that $\left(\operatorname{Der}(\log D), \operatorname{Der}_{\mathbb{C}}\left(\mathcal{O}_{X}\right)\right)$ is a Koszul pair is equivalent to saying that $D$ is a Koszul free divisor.

(1.5.2) Definition. 1) Let $L \subset L^{\prime}$ be a pair of Lie-Rinehart algebras over $(k, A)$. We say that $\left(L, L^{\prime}\right)$ is a pre-Spencer pair (over $\left.(k, A)\right)$ if the complex $\mathrm{U}\left(L^{\prime}\right) \stackrel{L}{\otimes} \mathrm{U}(L) A$ is cohomologically concentrated in degree 0 .

2) Similarly, we say that a pair $\left(\mathcal{L}, \mathcal{L}^{\prime}\right)$ of Lie algebroids over $(\mathcal{K}, \mathcal{A})$ is a preSpencer pair if $\left(\mathcal{L}_{p}, \mathcal{L}_{p}^{\prime}\right)$ is a pre-Spencer pair over $\left(\mathcal{K}_{p}, \mathcal{A}_{p}\right)$ for any $p \in M$, or equivalently, if the complex $\mathrm{U}\left(\mathcal{L}^{\prime}\right) \stackrel{L}{\otimes}_{\mathrm{U}(\mathcal{L})} \mathcal{A}$ is cohomologically concentrated in degree 0.

3) We say that $D$ is a pre-Spencer (free) divisor if $\left(\operatorname{Der}(\log D), \operatorname{Der}_{\mathbb{C}}\left(\mathcal{O}_{X}\right)\right)$ is a pre-Spencer pair over $\left(\mathbb{C}_{X}, \mathcal{O}_{X}\right)$.

From (3) and proposition (1.1.9) we know that

$$
\operatorname{Sp}_{L, L^{\prime}}=\mathrm{U}\left(L^{\prime}\right) \otimes_{\mathrm{U}(L)} \operatorname{Sp}_{L}(A)=\mathrm{U}\left(L^{\prime}\right) \stackrel{L}{\otimes}(L) A
$$

and the property for $\left(L, L^{\prime}\right)$ to be a pre-Spencer pair is equivalent to the fact that the complex $\mathrm{Sp}_{L, L^{\prime}}$ is cohomologically concentrated in degree 0 , and so it is a free resolution of $\mathrm{U}\left(L^{\prime}\right) / \mathrm{U}\left(L^{\prime}\right) \cdot L$ trough the augmentation (2). In particular, if $D$ is a Spencer divisor in the sense of [12], then it is a pre-Spencer divisor.

(1.5.3) Proposition. Let $L \subset L^{\prime}$ be a pair of Lie-Rinehart algebras over $(k, A)$. If $\left(L, L^{\prime}\right)$ is a Koszul pair over $A$ and $E$ is a left $\mathrm{U}(L)$-module flat 
(resp. free) over $A$, then the complex $\operatorname{Sp}_{L, L^{\prime}}(E)$ is a $\mathrm{U}\left(L^{\prime}\right)$-resolution (resp. a free $\mathrm{U}\left(L^{\prime}\right)$-resolution) of $\mathrm{U}\left(L^{\prime}\right) \otimes_{\mathrm{U}(L)} E$.

Proof. The proof is similar to the proof of proposition (1.1.9). We consider the filtration on the complex $\operatorname{Sp}_{L, L^{\prime}}(E)$ given by

$$
F^{i} \operatorname{Sp}_{L, L^{\prime}}^{-k}(E)=\left(F^{i-k} \mathrm{U}\left(L^{\prime}\right)\right) \otimes_{A} \bigwedge^{k} L \otimes_{A} E, i \geq 0
$$

Its graded complex is, by using the Poincaré-Birkhoff-Witt theorem, canonically isomorphic to the tensor product by $-\otimes_{A} E$ of the complex $\operatorname{Sym}_{A}\left(L^{\prime}\right) \otimes_{A}$ $\bigwedge^{\bullet} L$, where the differential is given by

$$
d^{-k}\left(P \otimes\left(\lambda_{1} \wedge \cdots \wedge \lambda_{k}\right)\right)=\sum_{i=1}^{k}(-1)^{i-1}\left(P \lambda_{i}\right) \otimes\left(\lambda_{1} \wedge \cdots \wedge \widehat{\lambda_{i}} \wedge \cdots \wedge \lambda_{k}\right)
$$

for $P \in \operatorname{Sym}_{A}\left(L^{\prime}\right), \lambda_{1}, \ldots, \lambda_{k} \in L$ and $k=1, \ldots, \mathrm{rk}_{A} L$.

Since $\left(L, L^{\prime}\right)$ is a Koszul pair and $E$ is flat over $A$, the complex

$$
\operatorname{Gr}_{F} \operatorname{Sp}_{L, L^{\prime}}(E)=\left(\operatorname{Sym}_{A}\left(L^{\prime}\right) \otimes_{A} \dot{\bigwedge} L\right) \otimes_{A} E
$$

is exact in degrees $\neq 0$, and so $\operatorname{Sp}_{L, L^{\prime}}(E)$ too, i.e. it is a resolution of its 0-cohomology $h^{0}\left(\operatorname{Sp}_{L, L^{\prime}}(E)\right)=\mathrm{U}\left(L^{\prime}\right) \otimes_{\mathrm{U}(L)} E$.

(1.5.4) Corollary. 1) Let $L \subset L^{\prime}$ be a pair of Lie-Rinehart algebras over $(k, A)$. If $\left(L, L^{\prime}\right)$ is a Koszul pair over $A$, then $\left(L, L^{\prime}\right)$ is a pre-Spencer pair. 2) In a similar way, any pair $\left(\mathcal{L}, \mathcal{L}^{\prime}\right)$ of Lie algebroids over $(\mathcal{K}, \mathcal{A})$ which is a Koszul pair over $\mathcal{A}$ is a pre-Spencer pair.

Proof. The second part follows straightforward from the first part, and the first part is a consequence of Proposition (1.5.3) in the case $E=A$.

(1.5.5) Proposition. Let $L \subset L^{\prime}$ be a pair of A-modules (resp. of LieRinehart algebras over $(k, A))$ which are $A$-free of finite rank. If $\left(L, L^{\prime}\right)$ is a Koszul pair over $A$ (resp. a pre-Spencer pair over $(k, A))$, then $\left(L[s], L^{\prime}[s]\right)$ is a Koszul pair over $A[s]$ (resp. a pre-Spencer pair over $(k[s], A[s])$ ).

Proof. It comes from the flatness of $k \rightarrow k[s]$ and the fact that $\operatorname{Sym}_{A[s]}(L[s])=$ $k[s] \otimes_{k} \operatorname{Sym}_{A}(L), \bigwedge_{A[s]} L[s]=k[s] \otimes_{k}\left(\bigwedge_{A} L\right)$ and

$$
\mathrm{U}_{(k[s], A[s])}\left(L^{\prime}[s]\right)=k[s] \otimes_{k} \mathrm{U}_{(k, A)}\left(L^{\prime}\right) .
$$




\subsection{The logarithmic Bernstein construction for free di- visors}

In the situation of section [1.3, let us suppose that $D$ is a free divisor and let us write $\mathcal{V}_{0}=\mathcal{D}_{X}(\log D)_{p}$. Since $D$ is free, the Lie-Rinehart algebra $\Theta_{f, s}$ defined in (77) is also $\mathcal{O}$-free of rank $n$.

Similar to the case of $\mathcal{D}[s]$, the filtered ring $\left(\mathcal{V}_{0}[s], F\right)$ is the enveloping algebra of the Lie-Rinehart algebra $\operatorname{Der}(\log D)_{p}[s]$ over $(\mathcal{O}[s], \mathbb{C}[s])$, and the filtered ring $\left(\mathcal{V}_{0}[s], F_{T}\right)$ is the enveloping algebra of the Lie-Rinehart algebra $F^{1} \mathcal{V}_{0}=\mathcal{O} \oplus \operatorname{Der}(\log D)_{p}$ over $(\mathcal{O}, \mathbb{C})$.

The free module of rank one over the ring $\mathcal{O}[s]$ generated by the symbol $f^{s}, \mathcal{O}[s] f^{s}$, has a natural left module structure over the ring $\mathcal{V}_{0}[s]$ : the action of a $\log$ arithmic derivation $\delta \in \operatorname{Der}(\log D)_{p}$ is given by $\delta\left(f^{s}\right)=\frac{\delta(f)}{f} s f^{s}$.

Let $\left\{\delta_{1}, \ldots, \delta_{n}\right\}$ be a basis of $\operatorname{Der}(\log D)_{p}$ and let us write $\delta_{i}(f)=\alpha_{i} f$ and $\eta_{i}=\sigma\left(\delta_{i}\right) \in \operatorname{Gr}_{F} \mathcal{V}_{0}=\mathcal{O}\left[\eta_{1}, \ldots, \eta_{n}\right]$ for $i=1, \ldots, n$. The $\zeta_{i}=\delta_{i}-\alpha_{i} s$, $i=1, \ldots, n$, form a basis of $\Theta_{f, s}$. Their symbols with respect to the total order filtration are

$$
\sigma_{T}\left(\zeta_{i}\right)=\eta_{i}-\alpha_{i} s \in \operatorname{Gr}_{F_{T}} \mathcal{V}_{0}[s]=\operatorname{Sym}_{\mathcal{O}}\left(F^{1} \mathcal{V}_{0}\right)=\mathcal{O}\left[s, \eta_{1}, \ldots, \eta_{n}\right]
$$

and so $\left(\Theta_{f, s}, F^{1} \mathcal{V}_{0}\right)$ is a Koszul pair. From corollary (1.5.4) we deduce that the complex $\mathrm{Sp}_{\Theta_{f, s}, F^{1} \mathcal{V}_{0}}$ is cohomologically concentrated in degree 0. On the other hand, a division argument and the existence of the unique expressions (5) show that $\operatorname{ann}_{\mathcal{V}_{0}[s]} f^{s}=\mathcal{V}_{0}[s] \Theta_{f, s}$. Finally, by using the augmentation (2) we obtain a proof of the following proposition.

(1.6.1) Proposition. Under the above conditions, the complex $\mathrm{Sp}_{\Theta_{f, s}, F^{1}} \mathcal{V}_{0}$ is a free resolution of the $\mathcal{V}_{0}[s]$-module $\mathcal{O}[s] f^{s}$.

(1.6.2) Proposition. If $D$ is Koszul free at $p$ then $\left(\Theta_{f, s}, F^{1} \mathcal{D}\right)$ is a Koszul pair over $\mathcal{O}$.

Proof. Let us take a system of local coordinates $x_{1}, \ldots, x_{n} \in \mathcal{O}$ and consider the symbols of the partial derivatives $\xi_{i}=\sigma\left(\frac{\partial}{\partial x_{i}}\right)$. With the notations above, we know that $\eta_{1}, \ldots, \eta_{n}$ form a regular sequence in $\operatorname{Gr}_{F} \mathcal{D}=$ $\operatorname{Sym}_{\mathcal{O}}\left(\operatorname{Der}_{\mathbb{C}}(\mathcal{O})\right)=\mathcal{O}\left[\xi_{1}, \ldots, \xi_{n}\right]$. Then, $s, \eta_{1}, \ldots, \eta_{n}$ form another regular sequence in $\mathcal{O}\left[s, \xi_{1}, \ldots, \xi_{n}\right]=\operatorname{Sym}_{\mathcal{O}}\left(F^{1} \mathcal{D}\right)$. Since the ideals $\left(s, \eta_{1}, \ldots, \eta_{n}\right)$ and $\left(s, \eta_{1}-\alpha_{1} s, \ldots, \eta_{n}-\alpha_{n} s\right)$ coincide, and we are dealing with homogeneous elements in graded rings, regular and quasi-regular sequence are the same (cf. [29], § 16) and $s, \eta_{1}-\alpha_{1} s, \ldots, \eta_{n}-\alpha_{n} s$ is also a regular sequence in $\mathcal{O}[s, \underline{\xi}]$. In particular, $\sigma_{T}\left(\zeta_{1}\right), \ldots, \sigma_{T}\left(\zeta_{n}\right)$ is a regular sequence in $\operatorname{Sym}_{\mathcal{O}}\left(F^{1} \mathcal{D}\right)$ and the proposition is proved. 
(1.6.3) Remark. Example (1.6.8) shows that, in the above proposition, the assumptions of being Koszul for $D$ is not necessary to have the Koszul property for $\left(\Theta_{f, s}, F^{1} \mathcal{D}\right)$.

The following theorem was announced in Remark 5.10, (a) in [7].

(1.6.4) Theorem. Let us suppose that $D$ is of differential linear type and Koszul free. Then the complex $\mathrm{Sp}_{\Theta_{f, s}, F^{1} \mathcal{D}}$ is a free resolution of the $\mathcal{D}[s]-$ module $\mathcal{D}[s] \cdot f^{s} \subset \mathcal{O}\left[f^{-1}, s\right] f^{s}$ and the canonical morphism



is an isomorphism.

Proof. From Proposition (1.6.1) and (3) we have

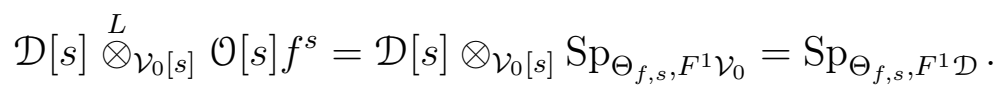

On the other hand, by Proposition (1.6.2), the complex $\operatorname{Sp}_{\Theta_{f, s}, F^{1} \mathcal{D}}$ is exact in degrees $\neq 0$. To conclude, we use that $D$ is of differential linear type:

$$
h^{0}\left(\operatorname{Sp}_{\Theta_{f, s}, F^{1} \mathcal{D}}\right)=\mathcal{D}[s] / \mathcal{D}[s] \cdot \Theta_{f, s}=\mathcal{D}[s] / \operatorname{ann}_{\mathcal{D}[s]} f^{s}=\mathcal{D}[s] \cdot f^{s} .
$$

(1.6.5) Remark. The hypotheses in the above theorem are satisfied in the case of locally quasi-homogeneous free divisors. In fact, following the lines in the proof of Theorem (3.2.6) and Theorem $4.1 \mathrm{in}$ [8, it is possible to deduce a weak (local) version of the logarithmic comparison theorem (LCT). Namely, under the hypothesis of theorem (1.6.4), there is a $k \gg 0$ such that the canonical morphism

$$
\mathcal{D} \stackrel{L}{\otimes} \mathcal{V}_{0}\left(\mathcal{O} \cdot f^{-k}\right) \rightarrow \mathcal{O}\left[f^{-1}\right]
$$

is an isomorphism in the derived category of $\mathcal{D}$-modules (see [7], Remark $5.10,(b))$. To go further and deduce a proof of the full version of the LCT for a locally quasi-homogeneous free divisors, one should prove before that that the Bernstein-Sato polynomial of its (reduced) equation does not have any integer root less than -1 . Unfortunately, we do not know any direct proof of this fact. Nevertheless, see [8], Th. 4.4 and Corollary (2.1.4) for other proofs of the LCT based on $D$-module theory.

The following remark has been pointed out by Torrelli (part [a] was also known by the authors). 
(1.6.6) Remark. Let $p$ be a point in $D, x_{1}, \ldots, x_{n} \in \mathcal{O}_{X, p}$ a system of local coordinates centered at $p$ and $f \in \mathcal{O}=\mathcal{O}_{X, p}$ a reduced local equation of $D$.

[a] We know that $f$ belongs to the integral closure of the gradient ideal $I=\left(f_{x_{1}}^{\prime}, \ldots, f_{x_{n}}^{\prime}\right)$ (cf. [43], $\left.\S 0.5,1\right)$ ), i.e there is an integer $d>0$ and elements $a_{i} \in I^{d-i}$ such that $f^{d}+a_{d-1} f^{d-1}+\cdots+a_{0}=0$. In particular, there is a homogeneous polynomial $F \in \mathcal{O}\left[s, \xi_{1}, \ldots, \xi_{n}\right]$ of degree $d>0$ such that $F\left(f, f_{x_{1}}^{\prime}, \ldots, f_{x_{n}}^{\prime}\right)=0$ and $F(s, 0, \ldots, 0)=s^{d}$. Let

$$
\delta_{i}=\sum_{j=1}^{n} a_{i j} \frac{\partial}{\partial x_{j}}, \quad 1 \leq i \leq m
$$

a system of generators of $\operatorname{Der}(\log D)_{p}$ and let us write $\delta_{i}(f)=\alpha_{i} f$. In other words, $\left(-\alpha_{i}, a_{i 1}, \ldots, a_{i n}\right), 1 \leq i \leq m$, is a system of generators of the syzygies of $f, f_{x_{1}}^{\prime}, \ldots, f_{x_{n}}^{\prime}$. If $D$ is of linear jacobian type at $p$, then the polynomial $F$ must be a linear combination of the polynomials

$$
-\alpha_{i} s+a_{i 1} \xi_{1}+\cdots+a_{i n} \xi_{n}, \quad 1 \leq i \leq m
$$

and making $\xi_{1}=\cdots=\xi_{n}=0$ we deduce that some of the $\alpha_{i}$ must be a unit, i.e. $f \in\left(f_{x_{1}}^{\prime}, \ldots, f_{x_{n}}^{\prime}\right)$. That shows that if $D=\{f=0\}$ is of linear jacobian type then $f$ is Euler homogeneous, i.e. there is a germ of vector field $\chi$ such that $\chi(f)=f$.

[b] Assume that the annihilator of $f^{-1}$ over $\mathcal{D}$ is generated by operators of order one. Then, from Proposition 1.3 of 44 we know that -1 is the smallest integer root of the Bernstein polynomial $b_{f}(s)$ of $f$. Reciprocally, let us assume that $D$ is of linear jacobian type at $p$ and that -1 is the smallest integer root of $b_{f}(s)$. Then, the annihilator of $f^{-1}$ over $\mathcal{D}$ is generated by operators of order one since it is obtained from the annihilator of $f^{s}$ over $\mathcal{D}[s]$ by making $s=-1$.

[c] Let us suppose now that $D$ is a free divisor which is Koszul and of differential linear type at $p$, and let $m_{0}$ be the smallest integer root of the Bernstein polynomial of $f$. For $l \geq-m_{0}$ the annihilator of $f^{-l}$ over $\mathcal{D}$ is generated by operators of order one, and the Koszul hypothesis allows us to apply Proposition 4.1 of [44] in order to obtain that $f$ is Euler homogeneous.

The following result is proved in [42], Cor. 3.12 in the polynomial case and has been independently pointed out to us by T. Torrelli.

(1.6.7) Proposition. If $D$ is of linear jacobian type and free at $p$, then it is Koszul free at $p$. 
Proof. Let $x_{1}, \ldots, x_{n} \in \mathcal{O}_{X, p}$ be a system of local coordinates centered at $p$ and $f \in \mathcal{O}=\mathcal{O}_{X, p}$ a reduced local equation of $D$. From remark (1.6.6), a) we know that $f$ is Euler homogeneous, i.e. $f \in\left(f_{x_{1}}^{\prime}, \ldots, f_{x_{n}}^{\prime}\right)$ and so $\operatorname{Jac}(D)_{p}=\left(f_{x_{1}}^{\prime}, \ldots, f_{x_{n}}^{\prime}\right)$.

Let $\left\{\delta_{i}=\sum_{j=1}^{n} a_{i j} \frac{\partial}{\partial x_{j}}\right\}_{1 \leq i \leq n}$ be a basis of $\operatorname{Der}(\log D)_{p}$ and let us write $\delta_{i}(f)=\alpha_{i} f$. Since $f$ is Euler homogeneous, we can take $\alpha_{1}=\cdots=\alpha_{n-1}=0$ and $\alpha_{n}=1$. In other words, $\left\{\left(a_{i 1}, \ldots, a_{i n}\right\}_{1 \leq i \leq n-1}\right)$ is a basis of the syzygies of $f_{x_{1}}^{\prime}, \ldots, f_{x_{n}}^{\prime}$.

Let $\theta: \mathcal{O}\left[\xi_{1}, \ldots, \xi_{n}\right] \rightarrow \mathcal{R}\left(\operatorname{Jac}(D)_{p}\right)=\mathcal{O}\left[f_{x_{1}}^{\prime} t, \ldots, f_{x_{n}}^{\prime} t\right]$ be the surjective map of $\mathcal{O}$-algebras defined by $\theta\left(\xi_{i}\right)=f_{x_{i}}^{\prime} t$. Since $\operatorname{Jac}(D)_{p}$ is an ideal of linear type, the kernel of $\theta$ is generated by the $\sigma\left(\delta_{i}\right)=\sum_{j=1}^{n} a_{i j} \xi_{j}, 1 \leq i \leq n-1$. So

$$
\operatorname{dim}\left(\frac{\mathcal{O}\left[\xi_{1}, \ldots, \xi_{n}\right]}{\left(\sigma\left(\delta_{1}\right), \ldots, \sigma\left(\delta_{n-1}\right)\right)}\right)=\operatorname{dim} \mathcal{R}\left(\operatorname{Jac}(D)_{p}\right)=n+1
$$

and $\sigma\left(\delta_{1}\right), \ldots, \sigma\left(\delta_{n-1}\right)$ is a regular sequence.

On the other hand if $F \sigma\left(\delta_{n}\right) \in\left(\sigma\left(\delta_{1}\right), \ldots, \sigma\left(\delta_{n-1}\right)\right)$, so $0=\theta(F) \theta\left(\sigma\left(\delta_{n}\right)\right)=$ $\theta(F) f t$ and we deduce that $F \in \operatorname{ker} \theta$ and $\sigma\left(\delta_{1}\right), \ldots, \sigma\left(\delta_{n}\right)$ is a regular sequence.

(1.6.8) Example. Let us suppose that $D \subset X$ is a non-necessarily free divisor and let $f=0$ be a reduced local equation of $D$ at a point $p \in D$. Let $\left\{\delta_{1}, \ldots, \delta_{m}\right\}$ be a system of generators of $\operatorname{Der}(\log D)_{p}$ and let us write $\delta_{i}(f)=\alpha_{i} f$.

Let us call $\operatorname{ann}_{\mathcal{D}[s]}^{(1)}\left(f^{s}\right)$ the ideal of $\mathcal{D}[s]$ generated by $\Theta_{f, s}(7)$ :

$$
\operatorname{ann}_{\mathcal{D}[s]}^{(1)}\left(f^{s}\right)=\mathcal{D}[s] \cdot\left(\delta_{1}-\alpha_{1} s, \ldots, \delta_{m}-\alpha_{m} s\right) \subset \operatorname{ann}_{\mathcal{D}[s]}\left(f^{s}\right) .
$$

The Bernstein functional equation for $f[3,21]$

$$
b(s) f^{s}=P(s) f^{s+1}
$$

means that the operator $b(s)-P(s) f$ belongs to the annihilator of $f^{s}$ over $\mathcal{D}[s]$. Then, an explicit knowledge of the ideal $\operatorname{ann}_{\mathcal{D}[s]}\left(f^{s}\right)$ allows us to find $b(s)$ by computing the ideal $\mathbb{C}[s] \cap\left(\mathcal{D}[s] \cdot f+\operatorname{ann}_{\mathcal{D}[s]}\left(f^{s}\right)\right)$. However, the ideal $\operatorname{ann}_{\mathcal{D}[s]}\left(f^{s}\right)$ is in general difficult to compute.

When $D$ is a divisor of differential linear type, $\operatorname{ann}_{\mathcal{D}[s]}\left(f^{s}\right)=\operatorname{ann}_{\mathcal{D}[s]}^{(1)}\left(f^{s}\right)$ and the computation of $b(s)$ is in principle easier. But there are examples of free divisors which are not of differential linear type for which the Bernstein polynomial $b(s)$ belongs to

$$
\mathbb{C}[s] \cap\left(\mathcal{D}[s] \cdot f+\operatorname{ann}_{\mathcal{D}[s]}^{(1)}\left(f^{s}\right)\right) .
$$


For instance, for $X=\mathbb{C}^{3}$ and $f=x_{1} x_{2}\left(x_{1}+x_{2}\right)\left(x_{1}+x_{2} x_{3}\right)$ (see Example 6.2 in [7]) or in the examples in page 445 of [13]. In all this examples the divisor is not Koszul, satisfies the logarithmic comparison theorem and $\left(\Theta_{f, s}, F^{1} \mathcal{D}\right)$ is a Koszul pair over $\mathcal{O}$ (see Prop. (1.6.2)).

\section{Integrable logarithmic connections with res- pect to a free divisor}

In this section we assume that $D \subset X$ is a free divisor.

A logarithmic connection with respect to $D$ is a locally $\mathcal{O}_{X}$-module $\mathcal{E}$ (the case where $\mathcal{E}$ is only supposed to be coherent certainly deserves to be studied, but it will not be treated in this paper) endowed with:

-) a $\mathbb{C}$-linear map (connection) $\nabla^{\prime}: \mathcal{E} \rightarrow \mathcal{E} \otimes_{\mathcal{O}_{X}} \Omega_{X}^{1}(\log D)$ satisfying $\nabla^{\prime}(a e)=$ $a \nabla^{\prime}(e)+e \otimes d a$, for any section $a$ of $\mathcal{O}_{X}$ and any section $e$ of $\mathcal{E}$,

or equivalently, with

-) a left $\mathcal{O}_{X^{-}}$-linear map $\nabla: \operatorname{Der}(\log D) \rightarrow \operatorname{End}_{\mathbb{C}_{X}}(\mathcal{E})$ satisfying the Leibniz rule $\nabla(\delta)(a e)=a \nabla(\delta)(e)+\delta(a) e$, for any logarithmic vector field $\delta$, any section $a$ of $\mathcal{O}_{X}$ and any section $e$ of $\mathcal{E}$.

The integrability of $\nabla^{\prime}$ is equivalent to the fact that $\nabla$ preserves Lie brackets. Then, we know from (1.1.7) and section 1.2 that giving an integrable logarithmic connection on a locally free $\mathcal{O}_{X}$-module $\mathcal{E}$ is equivalent to extending its original $\mathcal{O}_{X}$-module structure to a left $\mathcal{D}_{X}(\log D)$-module structure, and so integrable logarithmic connections are the same as left $\mathcal{D}_{X}(\log D)$-modules which are locally free of finite rank over $\mathcal{O}_{X}$.

Let us denote by $\mathcal{O}_{X}(\star D)$ the sheaf of meromorphic functions with poles along $D$. It is a holonomic left $\mathcal{D}_{X}$-module [21].

The first examples of integrable logarithmic connections (ILC for short) are the invertible $\mathcal{O}_{X}$-modules $\mathcal{O}_{X}(m D) \subset \mathcal{O}_{X}(\star D), m \in \mathbb{Z}$, formed by the meromorphic functions $h$ such that $\operatorname{div}(h)+m D \geq 0$.

If $f=0$ is a reduced local equation of $D$ at $p \in D$ and $\delta_{1}, \ldots, \delta_{n}$ is a local basis of $\operatorname{Der}(\log D)_{p}$ with $\delta_{i}(f)=\alpha_{i} f$, so $f^{-m}$ is a local basis of $\mathcal{O}_{X, p}(m D)$ over $\mathcal{O}_{X, p}$ and we have the following local presentation over $\mathcal{D}_{X, p}(\log D)$ (use (51))

$$
\mathcal{O}_{X, p}(m D) \simeq \mathcal{D}_{X, p}(\log D) / \mathcal{D}_{X, p}(\log D)\left(\delta_{1}+m \alpha_{1}, \ldots, \delta_{n}+m \alpha_{n}\right) .
$$

For any ILC $\mathcal{E}$ and any integer $m$, the locally free $\mathcal{O}_{X}$-modules $\mathcal{E}(m D):=$ $\mathcal{E} \otimes_{\mathcal{O}_{X}} \mathcal{O}_{X}(m D)$ and $\mathcal{E}^{*}:=\operatorname{Hom}_{\mathcal{O}_{X}}\left(\mathcal{E}, \mathcal{O}_{X}\right)$ are endowed with a natural structure of left $\mathcal{D}_{X}(\log D)$-module (cf. [8], $\left.\S 2\right)$, and they are again ILC, and the 
usual isomorphisms

$$
\mathcal{E}(m D)\left(m^{\prime} D\right) \simeq \mathcal{E}\left(\left(m+m^{\prime}\right) D\right), \quad \mathcal{E}(m D)^{*} \simeq \mathcal{E}^{*}(-m D)
$$

are $\mathcal{D}_{X}(\log D)$-linear.

\subsection{The logarithmic comparison problem}

If $D$ is Koszul free and $\mathcal{E}$ is an integrable logarithmic connection, then the complex $\mathcal{D}_{X} \stackrel{L}{\otimes}_{\mathcal{D}_{X}(\log D)} \mathcal{E}$ is concentrated in degree 0 and its 0-cohomology $\mathcal{D}_{X} \otimes_{\mathcal{D}_{X}(\log D)} \mathcal{E}$ is a holonomic $\mathcal{D}_{X}$-module (see [8], Prop. 1.2.3).

Let us denote by $\mathcal{D}_{X}(\star D)$ the sheaf of meromorphic linear differential operators with poles along $D$. One has obvious left and right $\mathcal{O}_{X}(\star D)$-linear isomorphisms

$$
\mathcal{O}_{X}(\star D) \otimes_{\mathcal{O}_{X}} \mathcal{D}_{X} \stackrel{\text { left }}{\simeq} \mathcal{D}_{X}(\star D) \stackrel{\text { right }}{\simeq} \mathcal{D}_{X} \otimes_{\mathcal{O}_{X}} \mathcal{O}_{X}(\star D)
$$

The induced maps

$$
\mathcal{O}_{X}(\star D) \otimes_{\mathcal{O}_{X}} \mathcal{D}_{X}(\log D) \rightarrow \mathcal{D}_{X}(\star D) \leftarrow \mathcal{D}_{X}(\log D) \otimes_{\mathcal{O}_{X}} \mathcal{O}_{X}(\star D)
$$

are also isomorphisms and so "meromorphic logarithmic linear differential operators" and "meromorphic linear differential operators" are the same:

$$
\mathcal{D}_{X}(\log D)(\star D)=\mathcal{D}_{X}(\star D) \text {. }
$$

If $\mathcal{E}$ is a left $\mathcal{D}_{X}(\log D)$-module, then the localization

$$
\mathcal{E}(\star D):=\mathcal{O}_{X}(\star D) \otimes_{\mathcal{O}_{X}} \mathcal{E}=\mathcal{D}_{X}(\star D) \otimes_{\mathcal{D}_{X}(\log D)} \mathcal{E}
$$

is a left $\mathcal{D}_{X}(\star D)$-module, and by scalar restriction, a left $\mathcal{D}_{X}$-module. Moreover, if $\mathcal{E}$ is a ILC, then $\mathcal{E}(\star D)$ is a meromorphic connection (locally free of finite rank over $\mathcal{O}_{X}(\star D)$ ) and then it is a holonomic $\mathcal{D}_{X}$-module (cf. [34], Th. 4.1.3). Actually, $\mathcal{E}(\star D)$ has regular singularities on the smooth part of $D$ (it has logarithmic poles! [16]) and so it is regular everywhere [33], Cor. 4.3-14, which means that if $\mathcal{L}$ is the local system of horizontal sections of $\mathcal{E}$ on $U=X-D$, the canonical morphism $\Omega_{X}^{\bullet}(\mathcal{E}(\star D)) \rightarrow R j_{*} \mathcal{L}$ is an isomorphism in the derived category.

For any ILC $\mathcal{E}$, or even for any left $\mathcal{D}_{X}(\log D)$-module (without any finiteness property over $\mathcal{O}_{X}$ ), one can define its logarithmic de Rham complex $\Omega_{X}^{\bullet}(\log D)(\mathcal{E})$ in the classical way, which is a subcomplex of $\Omega_{X}^{\bullet}(\mathcal{E}(\star D))$. It is clear that both complexes coincide on $U$. 
For any ILC $\mathcal{E}$ and any integer $m, \mathcal{E}(m D)$ is a sub- $\mathcal{D}_{X}(\log D)$-module of the regular meromorphic connection (and holonomic $\mathcal{D}_{X}$-module) $\mathcal{E}(\star D)$, and so we have a canonical morphism in the derived category of left $\mathcal{D}_{X^{-}}$ modules

$$
\rho_{\mathcal{E}, m}: \mathcal{D}_{X} \stackrel{L}{\otimes_{\mathcal{D}_{X}(\log D)}} \mathcal{E}(m D) \rightarrow \mathcal{E}(\star D),
$$

given by $\rho_{\varepsilon, m}\left(P \otimes e^{\prime}\right)=P e^{\prime}$.

Since $\mathcal{E}\left(m^{\prime} D\right)(m D)=\mathcal{E}\left(\left(m+m^{\prime}\right) D\right)$ and $\mathcal{E}\left(m^{\prime} D\right)(\star D)=\mathcal{E}(\star D)$, we can identify morphisms $\rho_{\mathcal{E}\left(m^{\prime} D\right), m}$ and $\rho_{\mathcal{E}, m+m^{\prime}}$.

We have the following theorem:

(2.1.1) Theorem. Let $\mathcal{E}$ be a ILC (with respect to the free divisor $D$ ) and let $\mathcal{L}$ be the local system of its horizontal sections on $U=X-D$. The following properties are equivalent:

1) The canonical morphism $\Omega_{X}^{\bullet}(\log D)(\mathcal{E}) \rightarrow R j_{*} \mathcal{L}$ is an isomorphism in the derived category of complexes of sheaves of complex vector spaces.

2) The inclusion $\Omega_{X}^{\bullet}(\log D)(\mathcal{E}) \hookrightarrow \Omega_{X}^{\bullet}(\mathcal{E}(\star D))$ is a quasi-isomorphism.

3) The morphism $\rho_{\mathcal{E}, 1}: \mathcal{D}_{X} \stackrel{L}{\otimes_{\mathcal{D}_{X}(\log D)}} \mathcal{E}(D) \rightarrow \mathcal{E}(\star D)$ is an isomorphism in the derived category of left $\mathcal{D}_{X}$-modules.

4) The complex $\mathcal{D}_{X} \stackrel{L}{\otimes_{\mathcal{D}_{X}(\log D)}} \mathcal{E}(D)$ is concentrated in degree 0 and the $\mathcal{D}_{X}$-module $\mathcal{D}_{X} \otimes_{\mathcal{D}_{X}(\log D)} \mathcal{E}(D)$ is holonomic and isomorphic to its localization along $D$.

5) The canonical morphism $j_{!} \mathcal{L}^{\vee} \rightarrow \Omega_{X}^{\bullet}(\log D)\left(\mathcal{E}^{*}(-D)\right)$ is an isomorphism in the derived category of complexes of sheaves of complex vector spaces.

Proof. The equivalence of the first three properties has been proved in [8], Th. 4.1.

The equivalence between 3) and 4) comes from the fact that the localization along $D$ of $\mathcal{D}_{X} \stackrel{L}{\otimes}_{\mathcal{D}_{X}(\log D)} \mathcal{E}(D)$ is canonically isomorphic to $\mathcal{E}(\star D)$ :

$$
\begin{aligned}
& {\left[\mathcal{D}_{X} \stackrel{L}{\otimes_{\mathcal{D}_{X}(\log D)}} \mathcal{E}(D)\right](\star D) \simeq \mathcal{D}_{X}(\star D) \otimes_{\mathcal{D}_{X}}\left[\mathcal{D}_{X} \stackrel{L}{\otimes_{\mathcal{D}_{X}(\log D)}} \mathcal{E}(D)\right] \simeq} \\
& \mathcal{D}_{X}(\star D) \otimes_{\mathcal{D}_{X}(\log D)} \mathcal{E}(D) \simeq \mathcal{O}_{X}(\star D) \otimes_{\mathcal{O}_{X}} \mathcal{E}(D) \simeq \mathcal{E}(D)(\star D) \simeq \mathcal{E}(\star D) .
\end{aligned}
$$

The equivalence between 5) and 1) is a consequence of the duality result in [8], Cor. 3.1.8,

$$
\Omega_{X}^{\bullet}(\log D)(\mathcal{E})^{\vee} \simeq \Omega_{X}^{\bullet}(\log D)\left(\mathcal{E}^{*}(-D)\right)
$$


[8], Cor. 3.1.6 and the fact that $\left(R j_{*} \mathcal{L}\right)^{\vee}=j_{!} \mathcal{L}^{\vee}$.

(2.1.2) Remark. In the above theorem, the complex $\mathcal{D}_{X} \stackrel{L}{\otimes_{\mathcal{D}_{X}(\log D)} \mathcal{E}(D)}$ need not to be holonomic even if its localization along $D$ is holonomic. For instance, in Example 5.1 of [8] for $\mathcal{E}=\mathcal{O}_{X}(-D)$ the $\mathcal{D}_{X}$-module $\mathcal{D}_{X} \otimes_{\mathcal{D}_{X}(\log D)}$ $\mathcal{O}_{X}$ is not holonomic. This fact has been also pointed out by Castro-Ucha and Torrelli. In particular, Problem 5.4 in loc. cit. has a negative answer.

For $D$ a locally quasi-homogeneous free divisor and $\mathcal{E}=\mathcal{O}_{X}$, the equivalent properties in Theorem (2.1.1) hold: this is the so called "logarithmic comparison theorem" [11, [8], Th. 4.4. Here we give a new proof using property 5) in Theorem (2.1.1),

(2.1.3) Proposition. Let us suppose that $D$ is a locally quasi-homogeneous (not necessarily free) divisor. Then the canonical morphism

$$
j ! \mathbb{C}_{U} \rightarrow \Omega_{X}^{\bullet}(\log D)\left(\mathcal{O}_{X}(-D)\right)
$$

is a quasi-isomorphism.

Proof. By Poincaré's lemma, the result is clear on $U$. To conclude, we apply [35], Lemma 3.3, (6) and we deduce that the complex $\Omega_{X}^{\bullet}(\log D)\left(\mathcal{O}_{X}(-D)\right)$ is acyclic at any point $p \in D$.

(2.1.4) Corollary. [11] Let $D$ be a locally quasi-homogeneous free divisor. Then the logarithmic comparison theorem holds:

$$
\Omega_{X}^{\bullet}(\log D) \stackrel{\sim}{\rightarrow} R j_{*} \mathbb{C}_{U} .
$$

Proof. The result is a straightforward consequence of Theorem (2.1.1) and Proposition (2.1.3).

\section{Main results}

Throughout this section, we suppose that $D \subset X$ is a free divisor and $\mathcal{E}$ is an ILC with respect to $D$.

\subsection{The Bernstein-Kashiwara construction for integrable logarithmic connections}

Let $p$ be a point in $D$ and $f \in \mathcal{O}=\mathcal{O}_{X, p}$ a reduced local equation of $D$. Let us write $\mathcal{D}=\mathcal{D}_{X, p}, \mathcal{V}_{0}=\mathcal{D}_{X}(\log D)_{p}, \operatorname{Der}(\log f)=\operatorname{Der}(\log D)_{p}$ and $E=\mathcal{E}_{p}$. 
We know that $\mathcal{E}(\star D)$ (resp. $E\left[f^{-1}\right]=\mathcal{E}(\star D)_{p}$ ) is a left $\mathcal{D}_{X}$-module (resp. a left $\mathcal{D}$-module) (see (8) ) .

The module $E\left[f^{-1}, s\right] f^{s}=E \otimes_{\mathcal{O}} \mathcal{O}\left[f^{-1}, s\right] f^{s}=E\left[f^{-1}\right] \otimes_{\mathcal{O}} \mathcal{O}\left[f^{-1}, s\right] f^{s}$ has a natural module structure over the ring $\mathcal{D}[s]$ : the action of a derivation $\delta \in \operatorname{Der}_{\mathbb{C}}(\mathcal{O})$ is given by $\delta\left(e f^{s}\right)=\delta(e) f^{s}+s \delta(f) f^{-1} e f^{s}$. We have $\mathcal{V}_{0}[s] \cdot E f^{s}=$ $E[s] f^{s}$, and so $E[s] f^{s}$ is a sub- $\mathcal{V}_{0}[s]$-module of $E\left[f^{-1}, s\right] f^{s}$.

From Proposition (1.1.9) we know that the complex $\operatorname{Sp}_{\operatorname{Der}(\log f)[s]}\left(E[s] f^{s}\right)$ is a free $\mathcal{V}_{0}[s]$-resolution of $E[s] f^{s}$ (here we consider $\mathcal{V}_{0}[s]$ as the enveloping algebra of the Lie-Rinehart algebra $\operatorname{Der}(\log f)[s]$ over $(\mathbb{C}[s], \mathcal{O}[s]))$. On the other hand, we have a canonical $\mathcal{D}[s]$-linear map

$$
P \otimes\left(e f^{s}\right) \in \mathcal{D}[s] \otimes_{\mathcal{V}_{0}[s]} E[s] f^{s} \mapsto P\left(e f^{s}\right) \in \mathcal{D}[s] \cdot E[s] f^{s} \subset E\left[f^{-1}, s\right] f^{s}
$$

inducing a surjective augmentation

$$
\rho_{E, s}: \mathcal{D}[s] \otimes_{\mathcal{V}_{0}[s]} \operatorname{Sp}_{D e r(\log f)[s]}^{0}\left(E[s] f^{s}\right) \rightarrow \mathcal{D}[s] \cdot\left(E[s] f^{s}\right)=\mathcal{D}[s] \cdot\left(E f^{s}\right) .
$$

The following theorem is strongly related to Theorem (1.6.4) in the case of the trivial ILC $\mathcal{E}=\mathcal{O}_{X}$.

(3.1.1) Theorem. Let us suppose that $D$ is of linear jacobian type at $p \in D$. Then the complex

$$
\mathcal{D}[s] \otimes_{\mathcal{V}_{0}[s]} \operatorname{Sp}_{\operatorname{Der}(\log f)[s]}\left(E[s] f^{s}\right)
$$

is exact and becomes a free $\mathcal{D}[s]$-resolution of $\mathcal{D}[s] \cdot\left(E f^{s}\right)$ through the map $\rho_{E, s}$ in (10).

Proof. From (3) and Propositions (1.5.3), (1.6.7) and (1.5.5), we deduce that the complex $\mathcal{D}[s] \otimes_{\mathcal{V}_{0}[s]} \operatorname{Sp}_{\operatorname{Der}(\log f)[s]}\left(E[s] f^{s}\right)$ is exact in degrees $\neq 0$. To conclude, we need to prove that the sequence

$$
\mathcal{D}[s] \otimes_{\mathcal{O}[s]} \operatorname{Der}(\log f)[s] \otimes_{\mathcal{O}[s]} E[s] f^{s} \stackrel{\varepsilon_{s}^{-1}}{\longrightarrow} \mathcal{D}[s] \otimes_{\mathcal{O}[s]} E[s] f^{s} \stackrel{\rho_{E, s}}{\longrightarrow} \mathcal{D}[s] \cdot\left(E f^{s}\right)
$$

is exact, where $\varepsilon_{s}^{-1}\left(P \otimes \delta \otimes\left(e f^{s}\right)\right)=(P \delta) \otimes\left(e f^{s}\right)-P \otimes \delta\left(e f^{s}\right)$ (see (1.1.8) and $\rho_{E, s}\left(P \otimes\left(e f^{s}\right)\right)=P\left(e f^{s}\right)$. The inclusion $\operatorname{Im} \varepsilon_{s}^{-1} \subset \operatorname{ker} \rho_{E, s}$ is clear.

Let $\left\{e_{1}, \ldots, e_{r}\right\}$ be an $\mathcal{O}$-basis of $E$. Any $Q \in \mathcal{D}[s] \otimes_{\mathcal{O}[s]} E[s] f^{s}$ can be uniquely written as $Q=\sum_{i=1}^{r} Q_{i} \otimes e_{i} f^{s}$ with $Q_{i} \in \mathcal{D}[s]$. We define the total order of $Q, \operatorname{deg}_{T}(Q)$, as the maximum of the orders of the $Q_{i}$ with respect to the total order filtration in $\mathcal{D}[s]$ and

$$
F_{T}^{k}\left(\mathcal{D}[s] \otimes_{\mathcal{O}[s]} E[s] f^{s}\right)=\left\{Q \mid \operatorname{deg}_{T}(Q) \leq k\right\} .
$$

Let $Q=\sum_{i=1}^{r} Q_{i} \otimes e_{i} f^{s} \in \operatorname{ker} \rho_{E, s}$. To prove that $Q$ belongs to the image of $\varepsilon_{s}^{-1}$, we proceed by induction on $\operatorname{deg}_{T}(Q)$. If $\operatorname{deg}_{T}(Q)=0$, then the $Q_{i}$ belong to $\mathcal{O}$ and the result is clear. Let us suppose now that

$$
F_{T}^{k-1}\left(\mathcal{D}[s] \otimes_{\mathcal{O}[s]} E[s] f^{s}\right) \cap \operatorname{ker} \rho_{E, s} \subset \operatorname{Im} \varepsilon_{s}^{-1}
$$


and $\operatorname{deg}_{T}(Q)=k$. We have

$$
0=\rho_{E, s}(Q)=\left(\sum_{i \in I} C_{Q_{i}, k} f^{-k} e_{i} f^{s}\right) s^{k}+\text { terms of lower degree in } s,
$$

where $I=\left\{i \mid \operatorname{deg}_{T}\left(Q_{i}\right)=k\right\}$ and $\varphi\left(\sigma_{T}\left(Q_{i}\right)\right)=C_{Q_{i}, k} t^{k}$ (see Lemma (1.3.1)). Consequently, $\sigma_{T}\left(Q_{i}\right) \in \operatorname{ker} \varphi$ for any $i \in I$ and, from Proposition (1.4.6) and Remark (1.4.3), there are $P_{i j} \in F_{T}^{k-1} \mathcal{D}[s]$ and $\gamma_{i j}=\delta_{i j}-\alpha_{i j} s \in \Theta_{f, s}$ such that

$$
\sigma_{T}\left(Q_{i}\right)=\sum_{j} \sigma_{T}\left(P_{i j}\right) \sigma_{T}\left(\gamma_{i j}\right), \quad \forall i \in I
$$

Let us consider

$$
Q^{\prime}=\sum_{i \in I}\left(\sum_{j} P_{i j} \otimes \gamma_{i j}\right) \otimes\left(e_{i} f^{s}\right) \in \mathcal{D}[s] \otimes_{\mathcal{O}[s]} \operatorname{Der}(\log f)[s] \otimes_{\mathcal{O}[s]} E[s] f^{s} .
$$

Since

$$
\begin{gathered}
\varepsilon_{s}^{-1}\left(Q^{\prime}\right)=\sum_{i \in I}\left(\sum_{j}\left(P_{i j} \gamma_{i j}\right)\right) \otimes\left(e_{i} f^{s}\right)-\sum_{i \in I}\left(\sum_{j} P_{i j} \otimes \gamma_{i j}\left(e_{i} f^{s}\right)\right)= \\
\sum_{i \in I}\left(\sum_{j}\left(P_{i j} \gamma_{i j}\right)\right) \otimes\left(e_{i} f^{s}\right)-\sum_{i \in I}\left(\sum_{j} P_{i j} \otimes\left(\delta_{i j} \cdot e_{i}\right) f^{s}\right),
\end{gathered}
$$

we have that $Q-\varepsilon_{s}^{-1}\left(Q^{\prime}\right) \in F_{T}^{k-1}\left(\mathcal{D}[s] \otimes_{\mathcal{O}[s]} E[s] f^{s}\right) \cap \operatorname{ker} \rho_{E, s}$, and by the induction hypothesis we obtain that $Q$ belongs to $\operatorname{Im} \varepsilon_{s}^{-1}$.

(3.1.2) Corollary. Under the hypothesis of Theorem (3.1.1), the canonical morphism

$$
\mathcal{D}[s] \stackrel{L}{\otimes} \mathcal{V}_{0}[s] E[s] f^{s} \rightarrow \mathcal{D}[s] \cdot\left(E f^{s}\right)
$$

is an isomorphism in the derived category of left $\mathcal{D}[s]$-modules.

Proof. It is a consequence of Proposition (1.1.9) and Theorem (3.1.1).

(3.1.3) Corollary. Let us suppose that D is a locally quasi-homogeneous free divisor. Then the complex

$$
\mathcal{D}[s] \otimes_{\mathcal{V}_{0}[s]} \operatorname{Sp}_{\operatorname{Der}(\log f)[s]}\left(E[s] f^{s}\right)
$$

is a free $\mathcal{D}[s]$-resolution of $\mathcal{D}[s] \cdot\left(E f^{s}\right)$.

Proof. It is a consequence of Theorems (3.1.1) and (1.4.4), and [6]. 


\subsection{The logarithmic comparison theorem}

Let us keep the notations of section 3.1. Let us also write $E\left[f^{-1}\right]=\mathcal{E}(\star D)_{p}$, $f^{-m} E=\mathcal{E}(m D)_{p}$ and

$$
\rho_{E, m}: \mathcal{D} \otimes_{\mathcal{O}}\left(f^{-m} E\right) \rightarrow E\left[f^{-1}\right]
$$

the induced map by $\rho_{\mathcal{E}, m}$ in (9), for any integer $m$.

(3.2.1) Lemma. There exists a non zero polynomial $b(s) \in \mathbb{C}[s]$ such that

$$
b(s) E f^{s} \subset \mathcal{D}[s] \cdot\left(E f^{s+1}\right) .
$$

Proof. Let $\left\{e_{1}, \ldots, e_{r}\right\}$ be an $\mathcal{O}$-basis of $E$ and let $b_{i}(s)$ be the BernsteinSato polynomial of $e_{i}$ considered as an element of the holonomic $\mathcal{D}$-module $E\left[f^{-1}\right]$. We take $b(s)=1$. c. m. $\left(b_{1}(s), \ldots, b_{r}(s)\right)$.

(3.2.2) Remark. a) The set of polynomials $b(s)$ in the above lemma is an ideal of $\mathbb{C}[s]$, whose monic generator will be denoted by $b_{E}(s)\left(\right.$ or $\left.b_{\mathcal{E}, p}(s)\right)$ and will be called Bernstein-Sato polynomial of $\mathcal{E}$ at $p$.

b) For any integer $k$ it is clear that the polynomials $b_{E}(s-k)$ satisfies $b_{E}(s-$ $k) E f^{s-k} \subset \mathcal{D}[s]\left(E f^{s-k+1}\right)$. In other words, $b_{\mathcal{E}, p}(s-k)=b_{\mathcal{E}(k D), p}(s)$. So, the polynomial $b_{l}(s)=\prod_{k=1}^{l} b_{E}(s-k)$ satisfies $b_{l}(s) E f^{s-l} \subset \mathcal{D}[s]\left(E f^{s}\right)$ and $E\left[f^{-1}\right]=\mathcal{D} \cdot\left(f^{-m} E\right)$, i.e. $\rho_{E, m}$ is surjective, if $b_{E}(s)$ has no integer roots less than $-m$.

The following proposition can be useful in order to compute the polynomial $b_{E}(s)$.

(3.2.3) Proposition. Let us suppose that $E$ is a cyclic $\mathcal{V}_{0}$-module generated by an element $e \in E$. Then, the polynomial $b_{E}(s)$ coincides with the Bernstein-Sato polynomial $b_{e}(s)$ of e with respect to $f$, where $e$ is considered as an element of the holonomic $\mathcal{D}$-module $E\left[f^{-1}\right]$.

Proof. For any $\delta \in \operatorname{Der}(\log f)$, we have

$$
\delta\left(e f^{s+1}\right)=(\delta e) f^{s+1}+(s+1) \frac{\delta(f)}{f} e f^{s+1}
$$

and so, since $\mathcal{V}_{0}=\mathcal{O}[\operatorname{Der}(\log f)]$, we deduce that $\left(\mathcal{V}_{0} \cdot e\right) f^{s+1} \subset \mathcal{V}_{0}[s] \cdot\left(e f^{s+1}\right)$ and

$$
\begin{gathered}
b_{E}(s) e f^{s} \in \mathcal{D}[s] \cdot\left(E f^{s+1}\right)=\mathcal{D}[s] \cdot\left(\left(\mathcal{V}_{0} \cdot e\right) f^{s+1}\right) \subset \\
\subset \mathcal{D}[s] \cdot\left(\mathcal{V}_{0}[s] \cdot\left(e f^{s+1}\right)\right)=\mathcal{D}[s] \cdot\left(e f^{s+1}\right) .
\end{gathered}
$$


In particular $b_{e}(s) \mid b_{E}(s)$. On the other hand,

$$
\begin{gathered}
b_{e}(s) E f^{s}=b_{e}(s)\left(\left(\mathcal{V}_{0} \cdot e\right) f^{s}\right) \subset b_{e}(s)\left(\mathcal{V}_{0}[s] \cdot\left(e f^{s}\right)\right)= \\
\mathcal{V}_{0}[s] \cdot\left(b_{e}(s) e f^{s}\right) \subset \mathcal{V}_{0}[s] \cdot\left(\mathcal{D}[s] \cdot\left(e f^{s+1}\right)\right) \subset \mathcal{D}[s] \cdot\left(E f^{s+1}\right)
\end{gathered}
$$

and so $b_{E}(s) \mid b_{e}(s)$.

\section{(3.2.4) Specialization at integers}

For each $m \in \mathbb{Z}$ and $r \geq 0$, let us denote by $\Phi_{m}: \mathcal{D}[s] \rightarrow \mathcal{D}, \Phi_{E, m}:$ $E\left[f^{-1}, s\right] f^{s} \rightarrow E\left[f^{-1}\right]$ and

$\Phi_{E, m}^{r}: \mathcal{D}[s] \otimes_{\mathcal{O}[s]}\left(\bigwedge^{r} \operatorname{Der}(\log f)[s]\right) \otimes_{\mathcal{O}[s]} E[s] f^{s} \rightarrow \mathcal{D} \otimes_{\mathcal{O}}\left(\bigwedge^{r} \operatorname{Der}(\log f)\right) \otimes_{\mathcal{O}}\left(f^{-m} E\right)$

the specialization maps making $s=-m$. It is clear that for any integer $m$ the following properties hold:

-) $\Phi_{E, m}\left(E[s] f^{s}\right) \subset f^{-m} E$,

-) $\Phi_{E, m}$ and the $\Phi_{E, m}^{r}$ are $\Phi_{m}$-linear,

-) $\Phi_{E, m} \circ \rho_{E, s}=\rho_{E, m} \circ \Phi_{E, m}^{0}$,

-) the $\Phi_{E, m}^{r}, r \geq 0$, commute with the differentials and define a morphism between Cartan-Eilenberg-Chevalley-Rinehart-Spencer complexes (see $(1.1 .8)$.

(3.2.5) Proposition. Under the above conditions, we have $\Phi_{E, k}^{0}\left(\operatorname{ker} \rho_{E, s}\right)=$ ker $\rho_{E, k}$ for all $k \geq-m_{0}$, where $m_{0}$ is the smallest integer root of $b_{E}(s)$.

Proof. Since $\Phi_{E, k} \circ \rho_{E, s}=\rho_{E, k} \circ \Phi_{E, k}^{0}$ for any integer $k$, we deduce that $\Phi_{E, k}^{0}\left(\operatorname{ker} \rho_{E, s}\right) \subset \operatorname{ker} \rho_{E, k}$.

Let $\left\{e_{1}, \ldots, e_{r}\right\}$ be an $\mathcal{O}$-basis of $E$ and $P=\sum_{i} P_{i} \otimes e_{i} f^{-k} \in \operatorname{ker} \rho_{E, k}$. Let us consider $P^{\prime}=\sum_{i} P_{i} \otimes e_{i} f^{s} \in \mathcal{D}[s] \otimes \mathcal{O}[s] E[s] f^{s}$. Since $\Phi_{E, k}\left(\rho_{E, s}\left(P^{\prime}\right)\right)=$ $\rho_{E, k}\left(\Phi_{E, k}^{0}\left(P^{\prime}\right)\right)=\rho_{E, k}(P)=0$ we deduce that $\rho_{E, s}\left(P^{\prime}\right)$ is divisible by $s+k$ in $E\left[f^{-1}, s\right] f^{s}$, i.e. there is a $B \in E[s] f^{s}$ and an $l>0$ such that $\rho_{E, s}\left(P^{\prime}\right)=$ $(s+k) f^{-l} B$.

From Remark (3.2.2), b), we know that $b_{l}(s) f^{-l} B \in \mathcal{D}[s]\left(E f^{s}\right)$ and so there is a $Q \in \mathcal{D}[s] \otimes_{\mathcal{O}[s]} E[s] f^{s}$ such that $b_{l}(s) f^{-l} B=\rho_{E, s}(Q)$. The element $R=b_{l}(s) P^{\prime}-(s+k) Q$ clearly belongs to ker $\rho_{E, s}$. If $k \geq-m_{0}, b_{l}(-k) \neq 0$ and

$$
P=\Phi_{E, k}^{0}\left(b_{l}(-k)^{-1} R\right) \in \Phi_{E, k}^{0}\left(\operatorname{ker} \rho_{E, s}\right)
$$


(3.2.6) Theorem. Let us suppose that $D$ is of linear jacobian type and let $m_{p}$ be the smallest integer root of $b_{\varepsilon, p}(s)$. Then, there is an open neighborhood $V$ of $p$ such that the restriction to $V$ of the morphism

$$
\rho_{\mathcal{E}, k}: \mathcal{D}_{X} \stackrel{L}{\otimes}_{\mathcal{D}_{X}(\log D)} \mathcal{E}(k D) \rightarrow \mathcal{E}(\star D)
$$

is an isomorphism in the derived category of $\mathcal{D}_{U}$-modules, for all $k \geq-m_{p}$.

Proof. By the coherence of the involved objects, we can work at the level of the stalks at $p$.

Since $D$ is Koszul (Prop. (1.6.7)], the complex

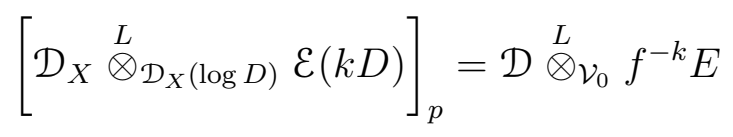

is exact in degrees $\neq 0$ (see Proposition (1.5.3)]. To conclude, we need to prove that the sequence

$$
\mathcal{D} \otimes_{\mathcal{O}} \operatorname{Der}(\log f) \otimes_{\mathcal{O}} f^{-k} E \stackrel{\varepsilon_{k}^{-1}}{\longrightarrow} \mathcal{D} \otimes_{\mathcal{O}} f^{-k} E \stackrel{\rho_{E, k}}{\longrightarrow} E\left[f^{-1}\right] \rightarrow 0
$$

is exact, where $\varepsilon_{k}^{-1}\left(P \otimes \delta \otimes\left(f^{-k} e\right)\right)=(P \delta) \otimes\left(f^{-k} e\right)-P \otimes \delta\left(f^{-k} e\right)$ (see $(1.1 .8)$ and $\rho_{E, k}\left(P \otimes\left(f^{-k} e\right)\right)=P\left(f^{-k} e\right)$.

From Remark (3.2.2), b), we know that $\rho_{E, k}$ is surjective if $k \geq-m_{p}$.

The inclusion $\operatorname{Im} \varepsilon_{k}^{-1} \subset \operatorname{ker} \rho_{E, k}$ is clear. For the opposite inclusion, we know by Proposition (3.2.5) that $\operatorname{ker} \rho_{E, k}=\Phi_{E, k}^{0}\left(\operatorname{ker} \rho_{E, s}\right)$ for any $k \geq-m_{p}$, and so from Theorem (3.1.1) we obtain

$$
\begin{aligned}
& \operatorname{ker} \rho_{E, k}=\Phi_{E, k}^{0}\left(\operatorname{ker} \rho_{E, s}\right)=\Phi_{E, k}^{0}\left(\operatorname{Im} \varepsilon_{s}^{-1}\right)= \\
& =\operatorname{Im}\left(\Phi_{E, k}^{0} \circ \varepsilon_{s}^{-1}\right)=\operatorname{Im}\left(\varepsilon_{k}^{-1} \circ \Phi_{E, k}^{1}\right) \subset \operatorname{Im} \varepsilon_{k}^{-1} .
\end{aligned}
$$

(3.2.7) Corollary. Let us suppose that $D$ is of linear jacobian type and let $\mathcal{L}$ be the local system of horizontal sections of $\mathcal{E}$ on $U=X-D$. Let $m_{p}$ be the smallest integer root of $b_{\mathcal{E}, p}(s)$. Then, there is an open neighborhood $V$ of $p$ such that the restriction to $V$ of the canonical morphism

$$
\Omega_{X}^{\bullet}(\log D)(\mathcal{E}(k D)) \rightarrow R j_{*} \mathcal{L}
$$

is an isomorphism in the derived category for $k \geq-m_{p}$.

Proof. It is a consequence of Theorems (2.1.1) and (3.2.6),

The above corollary answers a questions raised in [8], Ex. 5.3. 
(3.2.8) Corollary. With the same hypothesis as Corollary (3.2.7), let $m_{p}^{*}$ be the smallest integer root of $b_{\mathcal{E}^{*}, p}(s)$. Then, there is an open neighborhood $V$ of $p$ such that the restriction to $V$ of the canonical morphism

$$
j ! \mathcal{L} \rightarrow \Omega_{X}^{\bullet}(\log D)(\mathcal{E}(r D))
$$

is an isomorphism in the derived category for $r<m_{p}^{*}$.

Proof. It is a consequence of Corollary (3.1.8) in [8] and Corollary (3.2.7) applied to the dual connection $\mathcal{E}^{*}$.

(3.2.9) Remark. In Theorem (3.2.6) we obtain a global isomorphism $(V=X)$ if $m:=\inf _{p \in D} m_{p}>-\infty$ and $k \geq-m$. A similar remark applies to Corollaries (3.2.7) and (3.2.8).

(3.2.10) Remark. In the case $\mathcal{E}=\mathcal{O}_{X}$, Theorem (3.2.6) would give a proof of the LCT provided that the Bernstein-Sato polynomial of a reduced local equation of $D$ at any point has no integer roots less than -1 . This is the case when $D$ is locally quasi-homogeneous, but we do not know any direct proof of this fact (see Remark (1.6.5)].

(3.2.11) Remark. It would be interesting to have a proof of Theorem $(3.2 .6)$ by using property 5$)$ in Theorem (2.1.1), in a similar way as we did in Corollary (2.1.4) for the case of locally quasi-homogeneous free divisors and $\mathcal{E}=\mathcal{O}_{X}$.

(3.2.12) Remark. Let $X$ be a smooth algebraic variety over $\mathbb{C}$, or over a field of characteristic zero, and $D \subset X$ a hypersurface. The property of being free, Koszul free, of linear jacobian type or of differential linear type makes sense in the algebraic category, and there is an algebraic version of Theorem (3.2.6) whose proof seems possible following the lines in this paper.

\section{Applications to intersection $D$-modules}

In this section we assume that $D \subset X$ is a free divisor of linear jacobian type, and $\mathcal{E}$ is an ILC with respect to $D$.

Let $m_{p}\left(\right.$ resp. $\left.m_{p}^{*}\right)$ be the smallest integer root of $b_{\mathcal{E}, p}(s)\left(\right.$ resp. of $\left.b_{\mathcal{E}^{*}, p}(s)\right)$, and let us assume that

$$
m:=\inf _{p \in D} m_{p}>-\infty \quad \text { and } \quad m^{*}:=\inf _{p \in D} m_{p}^{*}>-\infty .
$$


Let $\mathcal{L}$ be the local system of the horizontal sections of $\mathcal{E}$ on $U=X-D$. As we saw in section 2.1 , the canonical morphism $\operatorname{DR} \mathcal{E}(\star D)=\Omega_{X}^{\bullet}(\mathcal{E}(\star D)) \rightarrow$ $R j_{*} \mathcal{L}$ is an isomorphism in the derived category. On the other hand, since $D$ is Koszul,

$$
\mathcal{D}_{X} \stackrel{L}{\otimes}_{\mathcal{D}_{X}(\log D)} \mathcal{E}(k D)=\mathcal{D}_{X} \otimes_{\mathcal{D}_{X}(\log D)} \mathcal{E}(k D),
$$

and from Theorem (3.2.6) we deduce that

$$
\operatorname{DR}\left(\mathcal{D}_{X} \otimes_{\mathcal{D}_{X}(\log D)} \mathcal{E}(k D)\right) \simeq \operatorname{DR} \mathcal{E}(\star D) \simeq \Omega_{X}^{\bullet}(\mathcal{E}(\star D)) \simeq R j_{*} \mathcal{L}
$$

for $k \geq-m$.

Let us consider now the dual local system $\mathcal{L}^{\vee}$, which appears as the local system of the horizontal sections of the dual ILC $\mathcal{E}^{*}$. Proceeding as above, we find that

$$
\operatorname{DR}\left(\mathcal{D}_{X} \otimes_{\mathcal{D}_{X}(\log D)} \mathcal{E}^{*}\left(k^{\prime} D\right)\right) \simeq \operatorname{DR} \mathcal{E}^{*}(\star D) \simeq \Omega_{X}^{\bullet}\left(\mathcal{E}^{*}(\star D)\right) \simeq R j_{*} \mathcal{L}^{\vee}
$$

for $k^{\prime} \geq-m^{*}$.

For $k+k^{\prime} \geq 1$ let us denote by

$$
\varrho_{\mathcal{E}, k, k^{\prime}}: \mathcal{D}_{X} \otimes_{\mathcal{D}_{X}(\log D)} \mathcal{E}\left(\left(1-k^{\prime}\right) D\right) \rightarrow \mathcal{D}_{X} \otimes_{\mathcal{D}_{X}(\log D)} \mathcal{E}(k D)
$$

the $\mathcal{D}_{X}$-linear map induced by the inclusion $\mathcal{E}\left(\left(1-k^{\prime}\right) D\right) \subset \mathcal{E}(k D)$, and by $\mathrm{IC}_{X}(\mathcal{L})$ the intersection complex of Deligne-Goresky-MacPherson associated with $\mathcal{L}$, which is described as the intermediate direct image $j_{\text {!* }} \mathcal{L}$, i.e. the image of $j_{!} \mathcal{L} \rightarrow R j_{*} \mathcal{L}$ in the category of perverse sheaves (cf. [2], Def. 1.4.22).

(4.1) Theorem. Under the above conditions, we have a canonical isomorphism in the category of perverse sheaves on $X$

$$
\mathrm{IC}_{X}(\mathcal{L}) \simeq \mathrm{DR}\left(\operatorname{Im} \varrho \varepsilon, k, k^{\prime}\right),
$$

for $k \geq-m, k^{\prime} \geq-m^{*}$ and $k+k^{\prime} \geq 1$. In other words, the "intersection $\mathcal{D}_{X^{-}}$ module" corresponding to $\mathrm{IC}_{X}(\mathcal{L})$ by the Riemann-Hilbert correspondence of Mebkhout-Kashiwara [23, [30, 31] is $\operatorname{Im} \varrho_{\varepsilon, k, k^{\prime}}$, for $k, k^{\prime} \gg 0$.

Proof. By using our duality results $([8], \S 3)$ and the Local Duality Theorem for holonomic $\mathcal{D}_{X}$-modules ([32], ch. I, Th. (4.3.1); see also [36]), we obtain

$$
\begin{gathered}
\operatorname{DR}\left(\mathcal{D}_{X} \otimes_{\mathcal{D}_{X}(\log D)} \mathcal{E}\left(\left(1-k^{\prime}\right) D\right)\right) \simeq \operatorname{DR}\left(\mathcal{D}_{X} \otimes_{\mathcal{D}_{X}(\log D)}\left(\mathcal{E}^{*}\left(k^{\prime} D\right)\right)^{*}(D)\right) \simeq \\
\operatorname{DR}\left(\mathbb{D}_{\mathcal{D}_{X}}\left(\mathcal{D}_{X} \otimes_{\mathcal{D}_{X}(\log D)} \mathcal{E}^{*}\left(k^{\prime} D\right)\right)\right) \simeq\left[\operatorname{DR}\left(\mathcal{D}_{X} \otimes_{\mathcal{D}_{X}(\log D)} \mathcal{E}^{*}\left(k^{\prime} D\right)\right)\right]^{\vee \stackrel{(12)}{\simeq}} \\
{\left[R j_{*} \mathcal{L}^{\vee}\right]^{\vee} \simeq j_{!} \mathcal{L} .}
\end{gathered}
$$


On the other hand, the canonical morphism $j_{!} \mathcal{L} \rightarrow R j_{*} \mathcal{L}$ corresponds, through the de Rham functor, to the $\mathcal{D}_{X}$-linear morphism $\varrho \varepsilon, k, k^{\prime}$, and the theorem is a consequence of the Riemann-Hilbert correspondence which says that the de Rham functor establishes an equivalence of abelian categories between the category of regular holonomic $\mathcal{D}_{X}$-modules and the category of perverse sheaves on $X$.

In [9] we use Theorem (4.1) to perform explicit computations in the case of locally quasi-homogeneous plane curves.

\section{References}

[1] A. G. Aleksandrov. Moduli of logarithmic connections along a free divisor. In Topology and geometry: commemorating SISTAG, volume 314 of Contemp. Math., pages 1-23. Amer. Math. Soc., Providence, RI, 2002.

[2] A. A. Beilinson, J. Bernstein, and P. Deligne. Faisceaux pervers, volume 100 of Astérisque. S.M.F., Paris, 1983.

[3] J. Bernstein. The analytic continuation of generalized functions with respect to a parameter. Funz. Anal. Appl., 6 (1972), 26-40.

[4] N. Bourbaki. Éléments de mathématique. Masson, Paris, 1980. Algèbre. Chapitre 10. Algèbre homologique.

[5] F. J. Calderón-Moreno. Logarithmic differential operators and logarithmic de Rham complexes relative to a free divisor. Ann. Sci. École Norm. Sup. (4), 32(5) (1999), 701-714. (math.AG/9807047).

[6] F. J. Calderón Moreno and L. Narváez Macarro. Locally quasihomogeneous free divisors are Koszul free. Proc. Steklov Inst. Math., 238 (2002), 72-77.

[7] F. J. Calderón-Moreno and L. Narváez-Macarro. The module $\mathcal{D} f^{s}$ for locally quasi-homogeneous free divisors. Compositio Math., 134(1) (2002), 59-74. (math.AG/0206262).

[8] F. J. Calderón Moreno and L. Narváez Macarro. Dualité et comparaison sur les complexes de de Rham logarithmiques par rapport aux diviseurs libres. Ann. Inst. Fourier (Grenoble), 55(1) (2005), 47-75. (math.AG/0411045). 
[9] F. J. Calderón Moreno and L. Narváez Macarro. Algebraic computation of some intersection D-modules. Lecture Notes in Computer Science, 4151 (2006), 132-143. (math.AG/0604287).

[10] H. Cartan and S. Eilenberg. Homological Algebra. Princeton Univ. Press, Princeton, N.J., 1956.

[11] F. J. Castro-Jiménez, D. Mond, and L. Narváez-Macarro. Cohomology of the complement of a free divisor. Trans. Amer. Math. Soc., 348 (1996), 3037-3049.

[12] F. J. Castro-Jiménez and J. M. Ucha-Enríquez. Free divisors and duality for D-modules. Proc. Steklov Inst. Math., 238 (2002), 88-96. (math.AG/0103085).

[13] F. J. Castro-Jiménez and J. M. Ucha-Enríquez. Testing the logarithmic comparison theorem for free divisors. Experiment. Math., 13(4) (2004), $441-449$.

[14] S. Chemla. A duality property for complex Lie algebroids. Math. Z., 232(2) (1999), 367-388.

[15] C. Chevalley and S. Eilenberg. Cohomology theory of Lie groups and Lie algebras. Trans. Amer. Math. Soc., 63 (1948), 85-124.

[16] P. Deligne. Equations Différentielles à Points Singuliers Réguliers, Lect. Notes in Math. 163, Springer-Verlag, Berlin-Heidelberg, 1970.

[17] H. Esnault and E. Viehweg. Logarithmic De Rham complexes and vanishing theorems. Invent. Math., 86 (1986), 161-194.

[18] J. C. Herz. Pseudo-algèbres de Lie. I. C. R. Acad. Sci. Paris, 236 (1953), 1935-1937.

[19] J. Huebschmann. Poisson cohomology and quantization. J. Reine Angew. Math., 408 (1990), 57-113.

[20] M. Kashiwara. Algebraic study of systems of linear differential equations. Master's thesis, Kyoto Univ., 1971. (In Japanese).

[21] M. Kashiwara. b-functions and holonomic systems. Invent. Math., 38 (1976), 33-53.

[22] M. Kashiwara. Algebraic study of systems of partial differential equations. Mém. Soc. Math. France (N.S.), vol. 63, 1995. (English translation of [20]). 
[23] M. Kashiwara. The Riemann-Hilbert problem for holonomic systems. Publ. Res. Inst. Math. Sci., 20(2) (1984), 319-365.

[24] K. Mackenzie. Lie groupoids and Lie algebroids in differential geometry, volume 124 of London Mathematical Society Lecture Note Series. Cambridge University Press, Cambridge, 1987.

[25] K. C. H. Mackenzie. General theory of Lie groupoids and Lie algebroids, volume 213 of London Mathematical Society Lecture Note Series. Cambridge University Press, Cambridge, 2005.

[26] Ph. Maisonobe and Z. Mebkhout. Le théorème de comparaison pour les cycles évanescents. In [27], pages 311-389, 2004.

[27] Ph. Maisonobe and L. Narváez Macarro (editors). Éléments de la théorie des systèmes différentiels géométriques, Séminaires et Congrès 8. Soc. Math. France, Paris, 2004. Cours du CIMPA, École d'été de Séville (1996).

[28] B. Malgrange. Cohomologie de Spencer (d'après Quillen). Cours à l'Univ. Orsay, 1967, unpublished.

[29] H. Matsumura. Commutative Ring Theory, volume 8 of Cambridge studies in advanced mathematics. Cambridge Univ. Press, Cambridge, 1986.

[30] Z. Mebkhout. Une équivalence de catégories. Comp. Math., 51 (1984), 51-62.

[31] Z. Mebkhout. Une autre équivalence de catégories. Comp. Math., 51 (1984), 63-88.

[32] Z. Mebkhout. Le formalisme des six opérations de Grothendieck pour les $\mathcal{D}_{X}$-modules cohérents, Travaux en cours 35. Hermann, Paris, 1989.

[33] Z. Mebkhout. Le théorème de positivité, le théorème de comparaison et le théorème d'existence de Riemann. In [27], pages 165-308, 2004.

[34] Z. Mebkhout and L. Narváez-Macarro. La théorie du polynôme de Bernstein-Sato pour les algèbres de Tate et de Dwork-MonskyWashnitzer. Ann. Sci. École Norm. Sup. (4), 24(2) (1991), 227-256.

[35] D. Mond. Differential forms on free and almost free divisors. Proc. London Math. Soc. (3), 81(3) (2000), 587-617. 
[36] L. Narváez Macarro. The Local Duality Theorem in D-module Theory. In [27], pages 59-88, 2004.

[37] R. S. Palais. The cohomology of Lie rings. In Proc. Sympos. Pure Math., Vol. III, pages 130-137. American Mathematical Society, Providence, R.I., 1961.

[38] D. G. Quillen. Formal properties of over-determined systems of linear partial differential equations. Harvard University, June 1964. Ph.D.

[39] G. S. Rinehart. Differential forms on general commutative algebras. Trans. Amer. Math. Soc., 108 (1963), 195-222.

[40] K. Saito. On the uniformization of complements of discriminant loci. Conference Notes, Amer. Math. Soc. Summer Institute, Williamstown, 1975.

[41] K. Saito. Theory of logarithmic differential forms and logarithmic vector fields. J. Fac. Sci. Univ. Tokyo, 27 (1980), 265-291.

[42] A. Simis. Differential idealizers and algebraic free divisors. In Commutative algebra, volume 244 of Lect. Notes Pure Appl. Math., pages 211-226. Chapman \& Hall/CRC, Boca Raton, FL, 2006.

[43] B. Teissier. Cycles évanescents, sections planes et conditions de Whitney. In Singularités à Cargèse (Rencontre Singularités Géom. Anal., Inst. Études Sci., Cargèse, 1972), pages 285-362. Astérisque, Nos. 7 et 8. Soc. Math. France, Paris, 1973.

[44] T. Torrelli. On meromorphic functions defined by a differential system of order 1. Bull. Soc. Math. France, 132 (2004), 591-612.

[45] W. V. Vasconcelos. Computational methods in commutative algebra and algebraic geometry, Algorithms and Computation in Mathematics, 2. Springer Verlag, New York, 1998.

[46] T. Yano. On the theory of b-functions. Publ. Res. Inst. Math. Sci., 14 (1978), 111-202.

Departamento de Álgebra, Facultad de Matemáticas, Universidad de Sevilla, P.O. Box 1160, 41080 Sevilla, Spain.

E-mail: \{calderon,narvaez\}@algebra.us.es 